\title{
Mechanical force-induced morphology changes in a human fungal pathogen
}

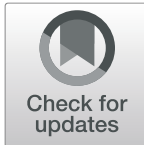

\author{
Charles Puerner ${ }^{1 \dagger}$, Nino Kukhaleishvili, ${ }^{1,}{ }^{\dagger}$, Darren Thomson ${ }^{1,3+}$, Sebastien Schaub ${ }^{1,4}$, Xavier Noblin $^{2 *}$, \\ Agnese Seminara ${ }^{2}$, Martine Bassilana ${ }^{1}$ and Robert A. Arkowitz ${ }^{1 *}$ (ID
}

\begin{abstract}
Background: The initial step of a number of human or plant fungal infections requires active penetration of host tissue. For example, active penetration of intestinal epithelia by Candida albicans is critical for dissemination from the gut into the bloodstream. However, little is known about how this fungal pathogen copes with resistive forces upon host cell invasion.

Results: In the present study, we have used PDMS micro-fabrication to probe the ability of filamentous C. albicans cells to penetrate and grow invasively in substrates of different stiffness. We show that there is a threshold for penetration that corresponds to a stiffness of $\sim 200 \mathrm{kPa}$ and that invasive growth within a stiff substrate is characterized by dramatic filament buckling, along with a stiffness-dependent decrease in extension rate. We observed a striking alteration in cell morphology, i.e., reduced cell compartment length and increased diameter during invasive growth, that is not due to depolarization of active $C d c 42$, but rather occurs at a substantial distance from the site of growth as a result of mechanical compression.

Conclusions: Our data reveal that in response to this compression, active Cdc42 levels are increased at the apex, whereas active Rho1 becomes depolarized, similar to that observed in membrane protrusions. Our results show that cell growth and morphology are altered during invasive growth, suggesting stiffness dictates the host cells that $C$. albicans can penetrate.
\end{abstract}

Keywords: Cell invasion, Mechanical force, Cell polarity, Cdc42, Cell morphology

\section{Background}

Polar tip growth, in which extension is limited to the apical surface, enables walled cells such as fungi and plants to explore their environment for nutrients and mating partners, while maintaining their surface to volume ratio [1]. Campas and Mahadevan [2] have derived simple scaling laws for cell geometry and identified a single dimensionless parameter that is

\footnotetext{
*Correspondence: xavier.noblin@unice.fr; arkowitz@unice.fr

${ }^{+}$Charles Puerner, Nino Kukhaleishvili, and Darren Thomson are co-first authors; order was determined by contribution to figures.

2Université Côte d'Azur, CNRS, Institute Physics of Nice (INPHYNI), Ave. J. Vallot, Nice, France

'Université Côte d'Azur, CNRS, INSERM, Institute of Biology Valrose (iBV), Parc Valrose, Nice, France

Full list of author information is available at the end of the article
}

sufficient to describe variation in the shape of tip growing cells using turgor pressure, cell wall elastic properties, and secretion rate. However, little is known with respect to the response of tip growing cells to mechanical stress. There are five fundamental types of mechanical stress: tension, compression, shear, torsion, and bending. Human and plant fungal pathogens can penetrate host tissue, and it is likely that they encounter compressive stress upon penetration and subsequent invasive growth. In addition to tissue penetration, growth within a spatially confined environment is critical for infection and successful dissemination of such fungal pathogens.

Fungal pathogens take advantage of different strategies to interact with their environment, of which tip

(c) The Author(s). 2020 Open Access This article is licensed under a Creative Commons Attribution 4.0 International License, which permits use, sharing, adaptation, distribution and reproduction in any medium or format, as long as you give appropriate credit to the original author(s) and the source, provide a link to the Creative Commons licence, and indicate if changes were made. The images or other third party material in this article are included in the article's Creative Commons licence, unless indicated otherwise in a credit line to the material. If material is not included in the article's Creative Commons licence and your intended use is not permitted by statutory regulation or exceeds the permitted use, you will need to obtain permission directly from the copyright holder. To view a copy of this licence, visit http://creativecommons.org/licenses/by/4.0/ The Creative Commons Public Domain Dedication waiver (http://creativecommons.org/publicdomain/zero/1.0/) applies to the data made available in this article, unless otherwise stated in a credit line to the data. 
growth is a common theme. Penetration of host tissue is critical for both human and plant fungal pathogens and requires not only the generation of sufficient force but also adhesion to the host cells to counter this force [3, 4]. Fungal pathogens have turgor pressures in the MPa range [3, 4], and for human fungal pathogens, this turgor pressure exceeds host cell resistance to penetration. Such host cells have elastic moduli that are in the $1-100 \mathrm{kPa}$ range [5-7], although the critical stress for material rupture is determinant. Both the human fungal pathogens Candida albicans [8] and Aspergillus fumigatus [9] can actively penetrate host tissue, which is a critical step in the infection process [10-14]. Previous studies have revealed that $C$. albicans can invade cells via hostinduced endocytosis and/or active penetration [8]. C. albicans invasion of the intestinal epithelia (small intestinal enterocytes) occurs almost exclusively by active penetration $[8,15,16]$, whereas both endocytosis and active penetration are important for invasion of the oral epithelia [17]. However, even with oral epithelia, at the early stages of infection, active penetration is the major route for tissue invasion [17]. Hence, a better understanding of active penetration should provide insight into the initial step of tissue damage for mucosal infections. Translocation of $C$. albicans through intestinal epithelial layers is facilitated by the fungal peptide toxin candidalysin [16, 18]. Previous studies have shown that $C$. albicans hyphal tips are asymmetrically positioned during growth on a stiff surface, i.e., a "nose down" morphology, and that perpendicular growth and contact to a stiff topographical ridge (less than the hyphal radius) results in an indentation of the ridge [19].

To investigate the relationship between substrate stiffness and $C$. albicans penetration and invasive growth, we have used micro-fabrication, together with time-lapse microscopy. We show that there is a threshold for penetration that corresponds to a stiffness of $\sim 200 \mathrm{kPa}$ and that invasive growth within a stiff substrate is characterized by dramatic filament buckling along with a stiffness-dependent decrease in extension rate. Nonetheless, a small percentage of cells are able to invade 200 $\mathrm{kPa}$ PDMS, suggesting that these cells may play a key role in infection, similar to that of the persister cells in biofilms. Furthermore, we observed a striking alteration in cell morphology during invasive growth, which is not due to depolarization of active Cdc42, but rather occurs at a substantial distance from the site of growth, as a result of mechanical forces. Our data reveal that in response to mechanical forces, C. albicans has increased active Cdc42 at the apex while active Rho1 is depolarized, similar to what is observed in PDGF-induced fibroblast membrane protrusions [20].

\section{Results}

Monitoring Candida albicans filamentous growth in micro-fabricated chambers

To investigate $C$. albicans hyphal growth, we took advantage of micro-fabrication approaches using the elastomer polydimethylsiloxane (PDMS) that, in particular, have been reported as single-cell force sensors for fission yeast cells [21]. We generated PDMS arrays with approximately $10^{5}$ microchambers, which were cylindrical in shape with a diameter of $10 \mu \mathrm{m}$, a depth of $5 \mu \mathrm{m}$, and $15 \mu \mathrm{m}$ spacing between adjacent chambers (Fig. 1a). C. albicans cells in micro-fabricated PDMS chamber arrays were visualized with inverted microscopes; imaging was carried out through an upright array of 150-200- $\mu$ m-thick PDMS. Figure 1b shows an $\mathrm{XZ}$ confocal reflectance scan through the PDMS microarray with the chambers and media at the top (highest position) and the coverslip below for support (a zoom of chambers is shown in Fig. 1c). C. albicans cells were mixed with fetal calf serum, added to the PDMS array, incubated for $\sim 1 \mathrm{~h}$, and subsequently, filamentous growth was followed over time (Fig. 1d). With lowstiffness PDMS (a high polymer to cross-linker ratio of 40:1) we observed two predominant filamentous growth modes: non-invasive growth on the PDMS surface and invasive growth within PDMS (Fig. 1e, f). By examination of the focal plane of the PDMS surface and the fungal filaments, using DIC optics, we were able to distinguish between non-invasive (surface) and invasive growth, referring to whether the filament tip is on or within the PDMS, respectively. Invasive growth was also confirmed by labeling the PDMS surface and filamentous cells (see below). Furthermore, we observed that the blastospore (round cell) portion of the filamentous cells, which grew in the microchambers, pushed back against the chamber wall upon PDMS filament penetration and the filament frequently buckled within PDMS, presumably due to the resistive force during growth within the elastomer (Figs. If and 2a). These results indicate that, in addition to having ideal optical properties, PDMS is compatible with C. albicans filamentous growth.

\section{Growth modes depend on substrate stiffness}

We followed C. albicans filamentous growth in PDMS of different stiffness, i.e. the extent to which an object resists deformation in response to an applied force, by varying the ratio of polymer to cross-linker. We observed two main growth modes from cells initially in chambers depending on PDMS stiffness: invasive growth, which predominated with less stiff PDMS (40:1) (Fig. 2a) and dramatic bending in the stiffer PDMS (30: 1) chambers, which was predominantly subapical (Fig. $2 b$, top panel). In contrast to Schizosaccharomyces pombe [21], extensive deformation of the chambers was not 


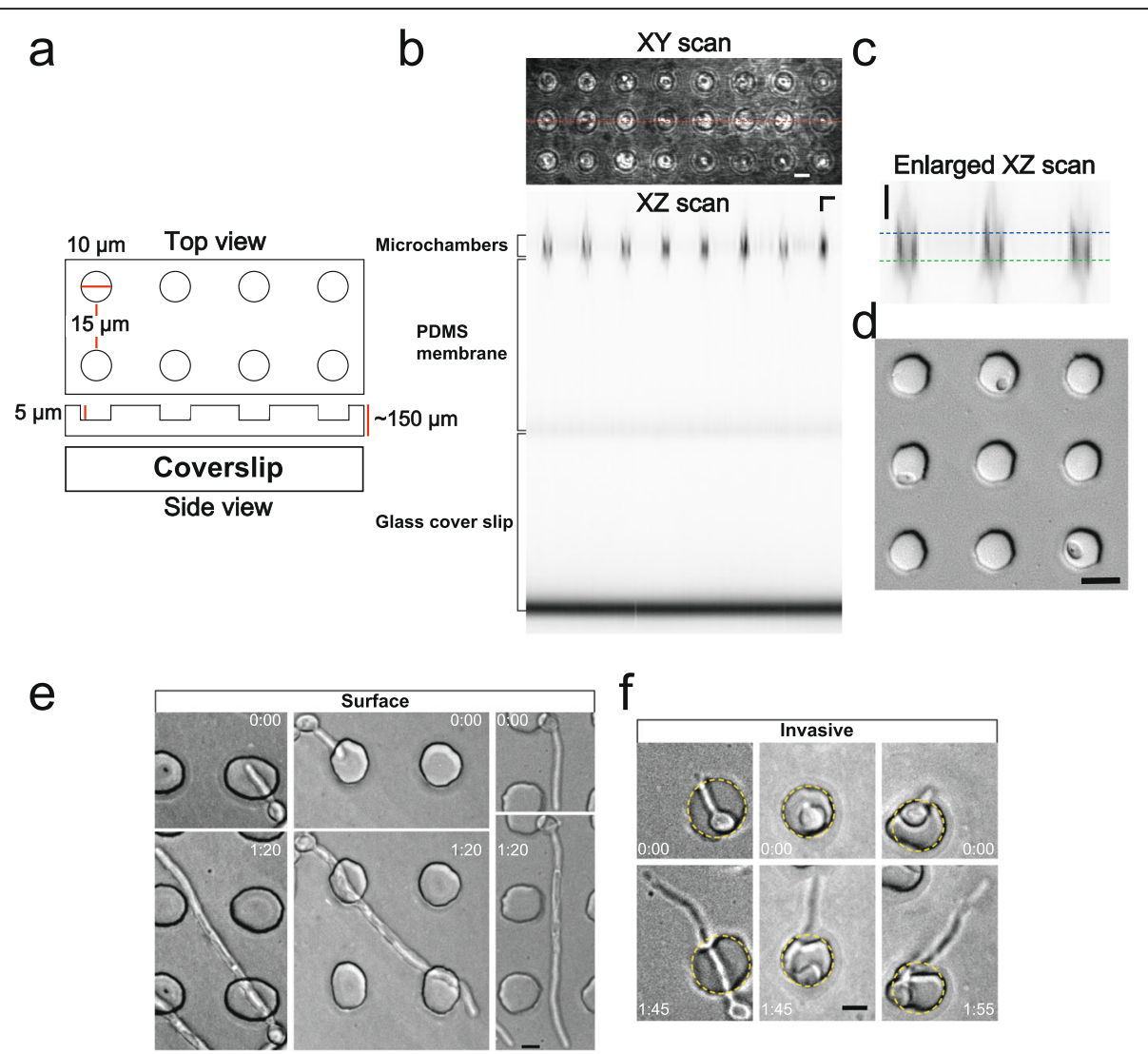

Fig. 1 Filamentous growth in and on PDMS. a Schematic of PDMS microchamber array. Top and side views are shown with dimensions indicated. b Characterization of PDMS microchamber array. XY transmission view (top) with the location of XZ confocal reflection scan (bottom) of upright PDMS mounted on a coverslip (indicated by dotted red line). Bars are $10 \mu \mathrm{m}$ in XY and XZ. c Enlarged XZ scan of PDMS microchambers. XZ confocal reflection microscopy of 3 microchambers with dotted lines, indicating top and bottom. Bar is $10 \mu \mathrm{m}$. $\mathbf{d}$ PDMS microchambers with C. albicans cells entrapped. C. albicans cells were mixed with serum (75\%) and added to PDMS microchamber arrays, and DIC image is shown. Note the cell with a germ tube (top row). Bar is $10 \mu \mathrm{m}$. e Non-invasive filamentous growth on PDMS micro-arrays. DIC images of growth on PDMS surface from 3 independent experiments on 40:1 PDMS to cross-linker, with indicated times (h:min). f Invasive filamentous growth within PDMS microarrays. DIC images of growth within PDMS from 2 independent experiments using 40:1 PDMS to cross-linker with times indicated. The initial PDMS chamber is outlined with a dotted yellow line to highlight deformation upon invasive filament extension

observed with C. albicans, which we attribute to the different sizes, geometries, and growth modes of these fungi; fission yeast has a radius of $\sim 2 \mu \mathrm{m}$, compared to C. albicans hyphal filaments with a radius of $\sim 1 \mu \mathrm{m}$ [22] (and our observations), resulting in a greater than 4-fold difference in the cross-sectional area. At most, a slight chamber deformation was observed with $C$. albicans, as cells frequently popped out of the chambers comprised of stiff PDMS or penetrated this material, when less stiff. Occasionally, at intermediate PDMS stiffness, we observed filamentous cells growing on the PDMS surface that appeared to be probing the surface with a "nose down" growth (Fig. 2b, bottom panel), as similarly observed [19]. This type of growth was suggestive of the filaments attempting to penetrate into PDMS and, consistent with this, we observed these filaments buckling and/or bending subapically at each attempt, prior to the tip popping out and forward. Buckling is defined as a sudden change in the shape of a component under load, i.e. change in the shape of the filament due to the physical forces it experiences. Subapical bending is, additionally, defined as a change in the direction of growth that results in curved filaments (Fig. 2c). In buckling, it is expected that the shape changes are largely reversed upon removal of the external forces, whereas in bending, the shape changes are not a result of the mechanical forces directly. Buckling can occur with a filament initially straight or bent/curved.

We next examined whether cells, initially in chambers, which were unable to invade, underwent bending. Figure $2 \mathrm{~d}$ shows that as the stiffness of PDMS increased (from 50:1 to 30:1 PDMS to cross-linker), there was an increase in the percentage of cells undergoing bending, concomitant with a decrease in those invading PDMS. During invasive growth, we also frequently observed buckling of the filament (Fig. 2a red arrowheads, c), i.e. a 
a

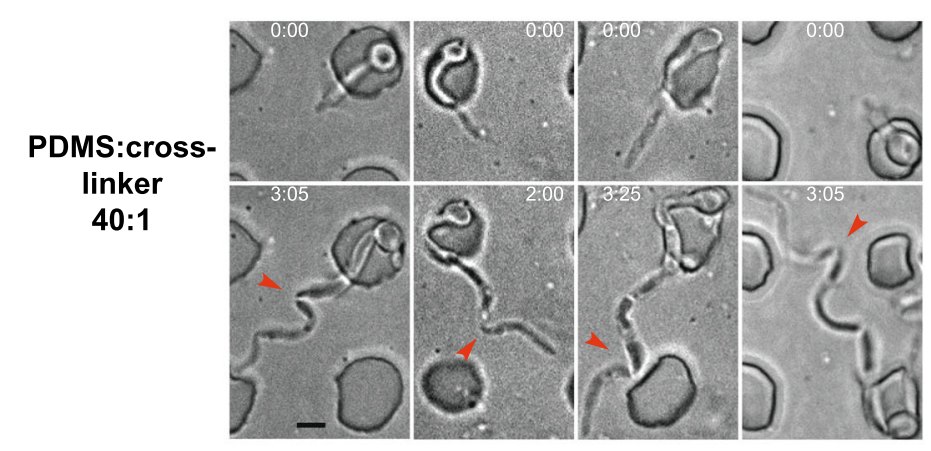

b

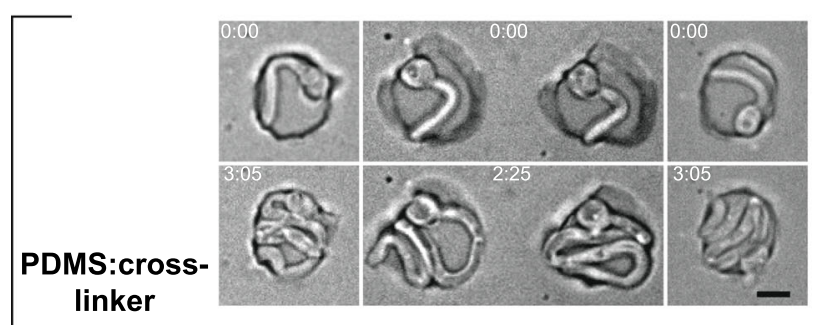

30:1

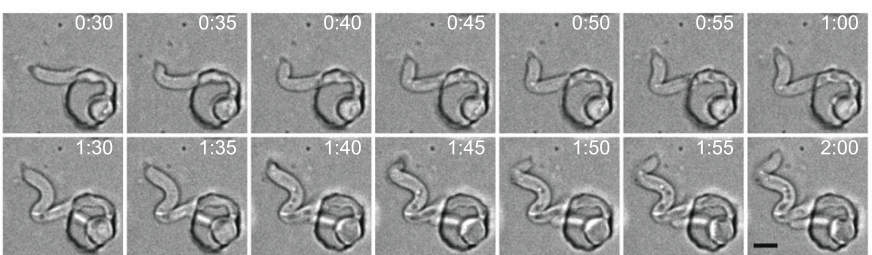

C

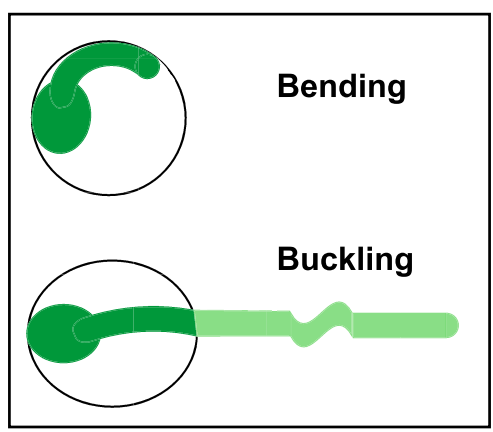

e

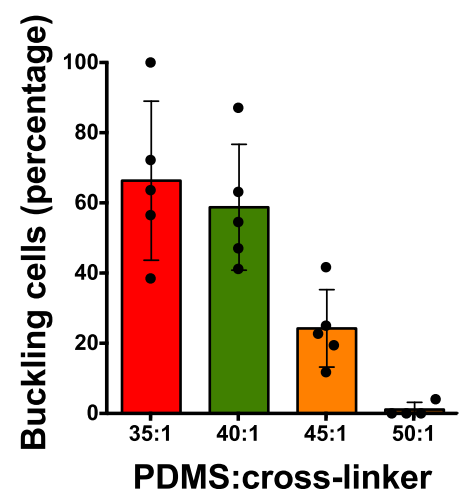

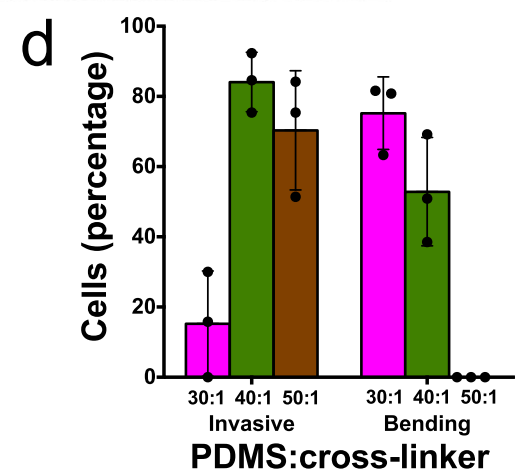

f

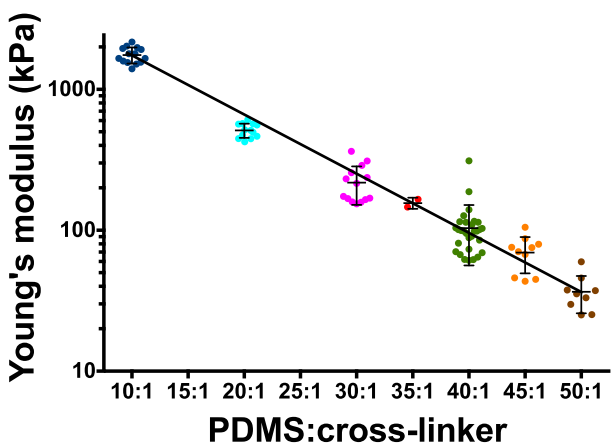

Fig. 2 (See legend on next page.) 


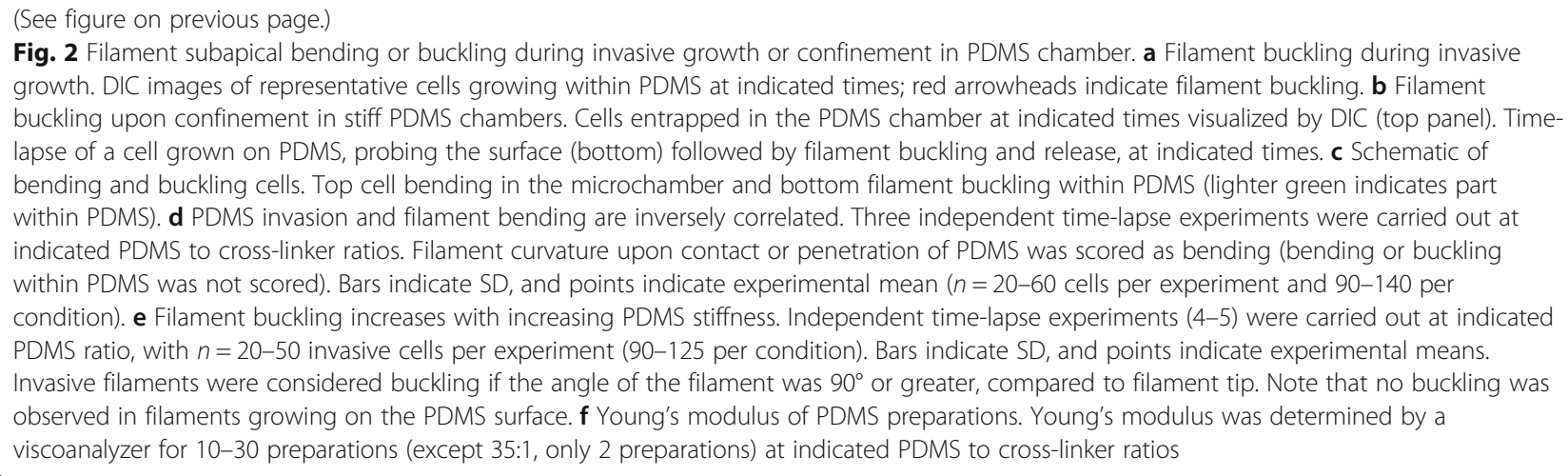

growth-dependent curvature that typically occurred at the portion of the filament within PDMS. Figure 2e shows that such buckling was dependent on the PDMS stiffness, with over half of invasive filaments buckling in the two stiffest PDMS (40:1 and 35:1).

To determine the mechanical properties of the different PDMS preparations, we used dynamic mechanical analysis for which measurements were reproducible over a range of PDMS stiffness. Oscillating strain at a frequency of 10 $\mathrm{Hz}$ was applied to PDMS samples, and stress $(\sigma)$-strain $(\varepsilon)$ curves were obtained (Additional file 1: Figure S1), from which Young's modulus was determined (initial $d \sigma / d \varepsilon$ ). Young's modulus is the quantitation of the stiffness, i.e. the ratio stress/strain for a uniaxial load, where stress is the force per unit area and strain is the proportional deformation (change in length divided by original length) and is dimensionless. Figure $2 \mathrm{f}$ shows the Young's modulus of different ratios of PDMS to cross-linker, which are in good agreement with published values [23-26]. The stiffness of lower ratio samples (10:1 and 20:1), intermediate ratios (30:1 to 40:1), and higher ratios (45:1 and 50:1) was similar to that of medical silicone implants [27], with a Young's modulus of $\sim 1 \mathrm{MPa}$; stiff tissues such as the myocardium [7], with a Young's modulus of $\sim 0.1-0.2$ $\mathrm{MPa}$; and less stiff tissues such as the epithelia [5, 6], with a Young's modulus of $\sim 40-70 \mathrm{kPa}$, respectively.

\section{Penetration into and escape from PDMS}

Given that active penetration is critical during the process of C. albicans epithelium invasion [10-12,14], we examined in further detail this process in PDMS. Figure 3a shows a filamentous cell that penetrates PDMS after 4 min (II; note that I, not shown, is prior to the filament contacting the chamber wall); subsequently grows invasively within PDMS (III); deforms the adjacent chamber (IV), resulting in a dramatic invagination; and exits PDMS into the adjacent well at 2:04 (V), followed by penetration into the opposing chamber at 2:08 (VI) and subsequent invasive growth (2:12; VII). The resistive force revealed by buckling of the filament, as well as deformation of the initial chamber during invasive growth (III), likely increases upon deformation and subsequent piercing into the adjacent well (IV), as the portion of the filament within PDMS buckled during this time (1:22-2:02), resulting in an S-shaped filament (Fig. 3a). The tension on the filament was released upon exiting PDMS into the adjacent well (V), as the tip of the filament appears to jump forward (2:04). The resistive force from the final step of growth (VII) also resulted in buckling of the filament (portion in the well) leading to an $M$ shape (2:42-3:00). This escape from PDMS is analogous, in some respects, to filaments bursting out of a macrophage [28-31]. Here, the filament pushes into a circle resulting in a deformation that does not require expansion of the surface area but rather local invagination of the chamber, which is easier to detect (Fig. 3a). Indeed, such a bursting out of PDMS was observed a number of times, and Additional file 1: Figure S2 shows such examples in different PDMS stiffness (40:1, $110 \mathrm{kPa} ; 35: 1,150 \mathrm{kPa}$; and 30: $1,250 \mathrm{kPa}$ ).

In order to better visualize the invasive growth within PDMS during these different steps, we followed cells in which GFP was targeted to the plasma membrane [32], by confocal spinning disk microscopy acquisition over a range of $z$-positions. Figure $3 \mathrm{~b}$ and $\mathrm{c}$ show a typical timelapse acquisition in which the analysis of the cell outline did not reveal a substantial change in the shape of the filament tip during invasive growth and bursting into the next well (Fig. 3c, d; Additional file 1: Figures S3A and S3B). Indeed, the radius of the curvature of the cell tip was identical to that of surface-growing cells, and there were no changes upon burst out of PDMS. Buckling of the filament was evident upon invasive growth and occurred over $35-45 \mathrm{~min}$, prior to the appearance of a septum (Fig. 3b (red arrowheads) and Fig. 4a, two examples). Analyses of the angle of the filament at which the septum formed ultimately, indicate that during invasive growth, cytokinesis occurs the majority of the time after the filament buckles (Fig. 4b). 


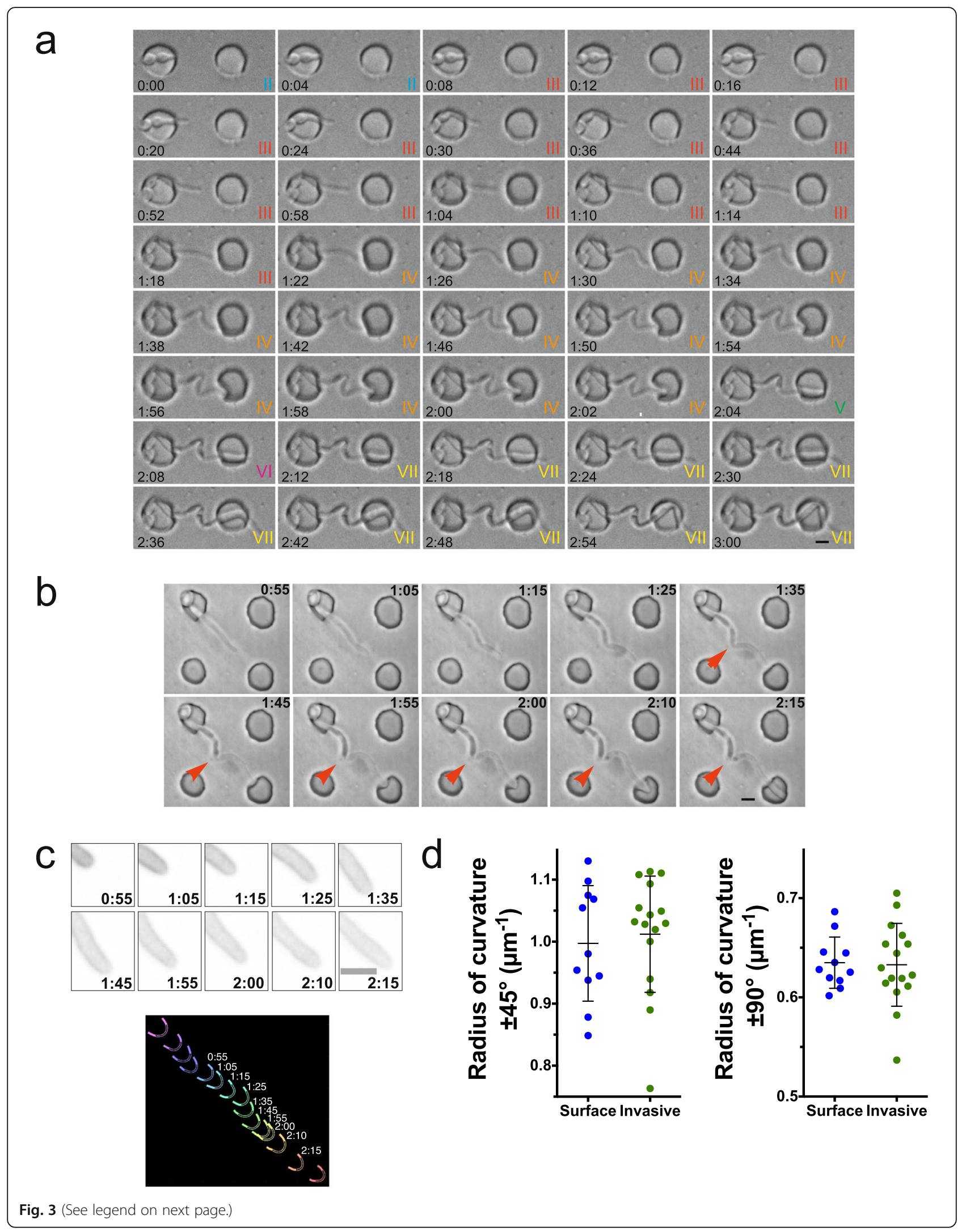


(See figure on previous page.)

Fig. 3 The filament tip shape is not substantially altered upon invasive growth and burst out. a Filament buckling and release upon invasive growth and penetration. Time-lapse experiment at PDMS to cross-linker ratio of 40:1 (Young's modulus $\sim 100 \mathrm{kPa}$ ) with DIC images acquired every 2 min. b, $\mathbf{c}$ Filament tip shape does not substantially change during PDMS burst out. b Typical time-lapse experiment at 40:1 PDMS to cross-linker ratio (Young's modulus $\sim 100 \mathrm{kPa}$ ) with DIC images every $5 \mathrm{~min}$. Red arrowheads indicate filament buckling. c Close up of sum projections of $13 \times 0.5 \mu \mathrm{m} z$-sections GFP-Ct $t_{\text {Rac } 1}$ fluorescent images from 3B (top), with tip curvature (bottom) at indicated times and $\pm 45^{\circ}$ curvature indicated by open lines and $\pm 90^{\circ}$ indicated by solid lines. $\mathbf{d}$ The radius of the curvature of invasive and surface growing cells is indistinguishable. The radius of the curvature is the average of $12-24 \times 5$ min time points ( $n=11-16$ cells) from 3 to 4 independent experiments for surface (PDMS ratio 40:1; Young's modulus $\sim 100 \mathrm{kPa}$ ) or invasively growing cells (with PDMS ratio 35:1-40:1; Young's modulus 150-100 kPa). Bars indicate SD

To analyze the physical constraints during penetration, invasive growth, and tip escape from the PDMS matrix, we established a physical experimental model, which consisted of a steel probe that mimics the filament shape, continuously advancing up to and into a cylinder of PDMS of different stiffness (Fig. 5a, b). The steel probe tip approximated the shape of the filament tip (Fig. 5c) with a radius of curvature $\left( \pm 45^{\circ}\right)$ of $1.1 \mu \mathrm{m}$ when normalized to the hyphal filament, compared to that of $1.0 \pm 0.1 \mu \mathrm{m}$ for the surface and invasively growing hyphal filaments. Figure $5 \mathrm{~d}$ shows the probe prior to PDMS rupture and subsequent to exiting from the PDMS. Experiments were carried out with a range of probe displacement rates encompassing that of the filament extension rates $(\sim 0.3 \mu \mathrm{m} / \mathrm{min}$ [33]), when scaled down to the filament diameter. Figure 6a shows an example of such a force versus displacement curve. The initial phase of increasing force corresponds to the elastic compression of PDMS ( $F_{\text {elast compr; }}$ analogous to growth stage II, Fig. 3a), culminating in the force required to break the PDMS surface, $F_{\text {crit }}$. The next phase corresponds to the extension within PDMS, analogous to invasive growth, $F_{\text {invas }}$ (analogous to growth stage III, Fig. 3a) culminating in PDMS exit ( $F_{\text {exiting; }}$ analogous to the end of growth stage IV, Fig. 3a). The final phase corresponds to when the end of the probe has emerged from the PDMS ( $F_{\text {out }}$; analogous to growth stage V, Fig. 3a). Figure 6b shows the $F_{\text {crit }}$ from the physical probe experiments as a function of PDMS stiffness. Filaments are able to penetrate PDMS of ratio 35:1, for which we measured $F_{\text {crit }}$ of $\sim 7 \mathrm{~N}$ with a 1-mm-diameter probe. Scaled to the diameter of a hyphal filament, this would correspond to a force of $\sim 31 \mu \mathrm{N}$, indicating that hyphal filaments generate forces larger than $31 \mu \mathrm{N}$ to penetrate PDMS. Scaling to the diameter of a hyphal filament was done assuming a constant critical stress, given the filament diameter is several orders of magnitude greater than the PDMS mesh size, and was calculated by taking the ratio of the metal probe/filament radius squared. Furthermore, our results indicate that at a stiffness of $200 \mathrm{kPa}$, for which little PDMS penetration is observed (Fig. 2d), the $F_{\text {crit }}$ is $\sim 8 \mathrm{~N}$ with a 1-mm-diameter probe, i.e. $\sim 35 \mu \mathrm{N}$ when scaled to a hyphal filament, which would correspond to the growth stalling force.

\section{Resistive force affects hyphal extension and morphology}

The buckling of the filaments, as well as the deformation of the PDMS wells during invasive growth, indicated that these filaments were responding to resistive force, whose magnitude we have measured in the physical model. The percentage of cells that penetrate PDMS is dependent on Young's modulus (Fig. 2d, f), and analyses of percent of PDMS invasion at two stiffness values indicate that the threshold for invasion is between 120 and $200 \mathrm{kPa}$ (Fig. 7a). Hence, to investigate the effects of resistive force on filamentous growth, we determined the length of the filaments over time from cells growing on and within PDMS in this range of stiffness. Figure $7 b$ shows that cells extend at a constant rate, which is reduced by $\sim 30 \%$ within PDMS from an average of $0.28 \pm$ $0.07 \mu \mathrm{m} / \mathrm{min} \quad(n=29)$ for surface growth to $0.19 \pm$ $0.05 \mu \mathrm{m} / \mathrm{min} \quad(n=32)$ when filaments were growing within PDMS with a stiffness of $\sim 100 \mathrm{kPa}$ (Fig. 7c). This filament extension rate was further reduced to $0.15 \pm$ $0.08 \mu \mathrm{m} / \mathrm{min}(n=23)$ upon growth in PDMS with a stiffness of $\sim 150 \mathrm{kPa}$ (Fig. 7c). To confirm that the reductions in extension rate during invasive growth were not due to the substantial changes in the $z$-position of the growing filament apex, cells were grown in PDMS chambers that had been stained with fluorescent ConA and $z$ section images were projected onto the $\mathrm{XZ}$ plane (Fig. 7d). These projections show that the filaments grow slightly downward in PDMS, below the bottom of the chamber, with maximally $5 \mu \mathrm{m}$ displacement in the $z$ axis for a $25-\mu \mathrm{m}$ filament, resulting in at most a $2 \%$ reduction in extension rate upon projection in the $\mathrm{XY}$ plane. In contrast, Fig. 8a shows that the mean filament extension rate of cells grown on the surface is not dependent on the substrate's stiffness, with indistinguishable rates on PDMS with Young's modulus from 100 to $200 \mathrm{kPa}$. Furthermore, the extension rate of invasive growth normalized for that of surface growth from each experiment correlates with substrate stiffness (Fig. 8b). Extrapolation to the $y$-intercept, where the invasive extension rate, equals the surface rate indicates a substrate stiffness of $\sim 20 \mathrm{kPa}$, suggesting that during filamentous growth on PDMS, the cells experience this resistive force from adhesion. Consistently, the surface extension rate was slightly reduced compared to that in 


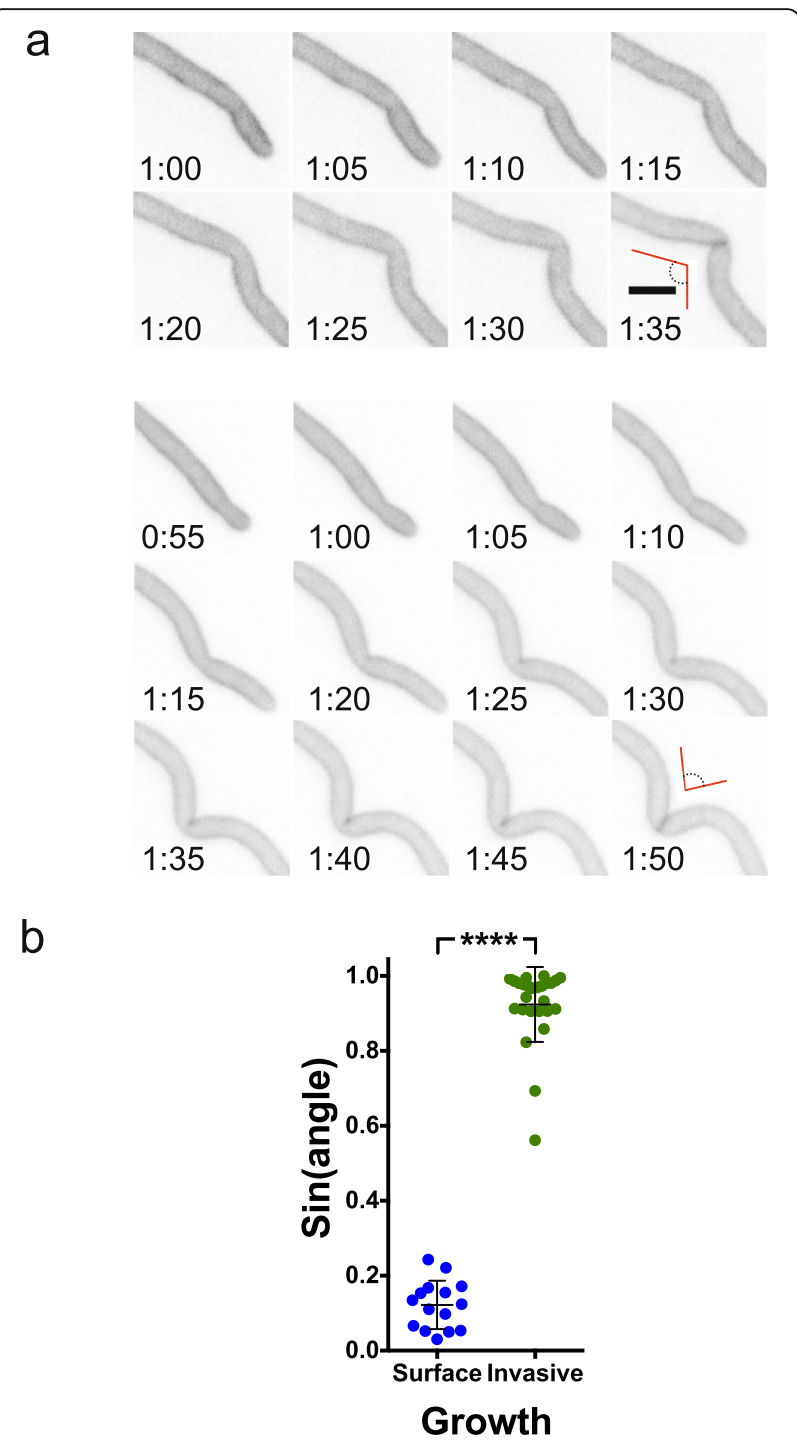

Fig. 4 Cell division occurs at the site of filament buckling during invasive growth. a Examples (upper and lower panels) of time-lapse images (GFP sum projections) during invasive growth in 40:1 PDMS ratio, showing cell division site at the location of filament buckling with red lines highlighting the buckling angle. b Cell division site occurs where the filament is buckled. Cells were grown either invasively within PDMS (40:1; Young's modulus $~ 100 \mathrm{kPa}$ ) or on the surface of PDMS (30:1-40:1; Young's modulus 250-100 kPa), $n=26$ and 15 , respectively. GFP sum projections were analyzed over time from 5 independent experiments and Sin of angle when the septum formed is shown. Septum formation was observed on average $47 \pm$ 20 min after buckling was evident in invasive cells. Bars indicate SD with $p<0.0001$

liquid media $(0.26 \pm 0.09 \mu \mathrm{m} / \mathrm{min}$ compared to $0.32 \pm$ $0.01 \mu \mathrm{m} / \mathrm{min} ; p=0.001$ ) (Fig. 8a). Of note, very few cells invaded PDMS at $200 \mathrm{kPa}$, on average $\sim 5 \%$ (Fig. 7a), but, strikingly, the filament extension rate for these "escapers" was similar to that of less stiff PDMS, raising the attractive possibility that these cells may play a critical role in tissue invasion.
The difference in filament extension rate could either be due to an overall reduction in cell growth or a reduction in polarized, apical growth. To differentiate between these possibilities, we determined the length and diameter of compartments (between 2 septa) for cells growing on the surface and within PDMS $(100 \mathrm{kPa})$. Figure $9 \mathrm{a}$ and $\mathrm{b}$ show that the compartment length decreased $\sim 30 \%$, from $24.6 \pm 3.0 \mu \mathrm{m}$ $(n=100)$ for surface growing cells to $16.6 \pm 1.8 \mu \mathrm{m}$ $(n=120)$ during invasive growth, and the filament diameter increased concomitantly from $2.1 \pm 0.2 \mu \mathrm{m}$ for surface growing cells to $2.5 \pm 0.2 \mu \mathrm{m}$ during invasive growth. As a result, the compartment volume remained constant $\left(83 \pm 17 \mu \mathrm{m}^{3}\right.$ for surface growing cells compared to $80 \pm 18 \mu \mathrm{m}^{3}$ for invasively growing cells), indicative of altered polarized growth. Consistently, analyses of filamentous cells grown within stiffer PDMS $(150 \mathrm{kPa})$ revealed a further decrease in compartment length $(14.6 \pm 2.5 \mu \mathrm{m})$ and an increased diameter $(2.8 \pm 0.3 \mu \mathrm{m})$. This altered morphology is dependent on growth against a resistive force in PDMS as the diameter in the part of the filament outside PDMS was similar to that of surface growing cells (Fig. 10a). In addition to the comparison of cells growing on the surface and within PDMS, we examined the relatively rare occurrence of cells transitioning between these growth modes. Figure 10b and c show an example of such a transition, in which the extension rate is reduced during invasive growth in PDMS and increases exiting PDMS. Measurements of the filament diameter just before and after the filament exited PDMS revealed an increased filament diameter during invasive growth that was significantly reduced upon exiting PDMS $(2.7 \pm 0.2 \mu \mathrm{m}$ compared to $2.3 \pm 0.3 \mu \mathrm{m}$, Fig. $10 \mathrm{~d})$.

Reduced filament extension rate in response to a resistive force suggested that similar effects could be observed in cells undergoing non-invasive, dramatic subapical bending in chambers of stiff PDMS (Additional file 1: Figure S4A). Additional file 1: Figure S4B shows that, in such conditions, there was indeed a dramatic reduction in filament extension rate with an average (over $100-150 \mathrm{~min}$ ) of $0.10 \pm$ $0.01 \mu \mathrm{m} / \mathrm{min}$. Surface growth rates were constant over time, whereas extension rates of cells undergoing dramatic subapical bending decreased concomitantly with the cell filling the well (Additional file 1: Figure S4C); initial rates of extension were 3 -fold reduced from surface growth, and these were further reduced 3-fold after $2 \mathrm{~h}$ of growth. Due to the complex geometries during such a growth mode, we were unable to determine the resistive force that the filament experiences while it fills up the chamber; however, the initial extension rate is similar to that of filaments growing invasively in $150 \mathrm{kPa}$ PDMS. 


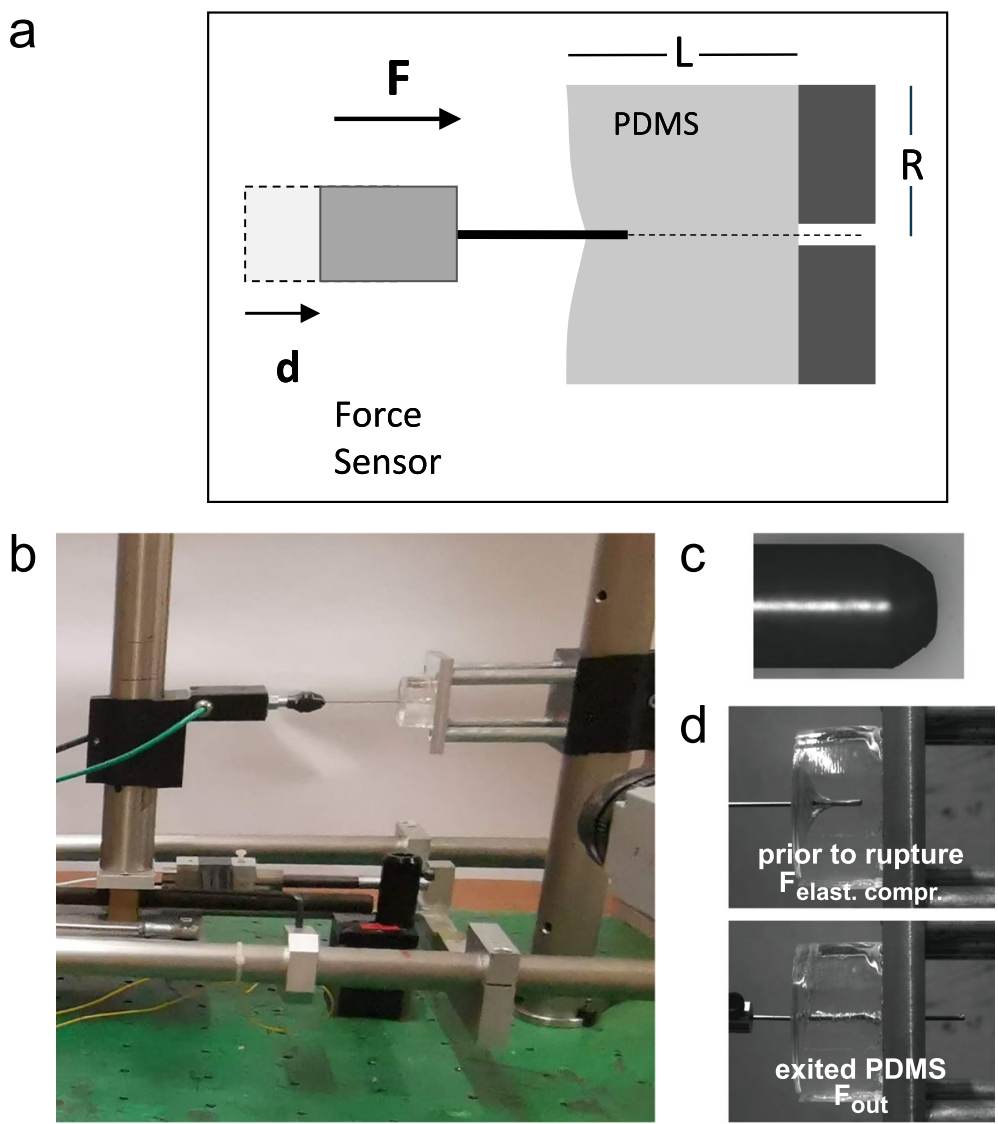

Fig. 5 Physical model of PDMS invasive growth. a Schematic of the physical model. The distance probe traveled is $d ; F$ is the force detected by the sensor; $L$ is the PDMS length, and $R$ is the PDMS radius. $\mathbf{b}$ Image of the physical model setup. Force sensor attached to 1-mm-diameter probe (left) and PDMS on the right as indicated in a. c Shape of the steel probe tip. Probe is $1 \mathrm{~mm}$ diameter. d PDMS penetration. PDMS (40:1; Young's modulus $\sim 100 \mathrm{kPa}$ ) cylinder (1.75 cm length and radius) with $1 \mathrm{~mm}$ probe displacement of $1.6 \mu \mathrm{m} / \mathrm{s}$. Upper image, prior to rupture of PDMS; lower image, probe exit from PDMS

\section{Determination of the effective turgor pressure}

From the comparison of extension rates within PDMS of different stiffness (Fig. 7c), we determined the effective turgor pressure in C. albicans hyphae, using the viscoplastic growth model [21]. This determination makes use of $F_{\text {invas }}$ values, measured from the physical model (Fig. 6), after scaling these forces from a cylinder with a radius of $0.5 \mathrm{~mm}$ to that of $1.04 \mu \mathrm{m}$. In order to correctly extrapolate the macroscopic measurements to the microscopic scale of filamentous cells, we analyzed the physical forces at play. The mode of extension during hyphal growth and in this physical experimental model is different, as new material is incorporated into the hyphal tip, i.e. growth occurs via apical extension, whereas in the physical experimental model, the probe is pushed into the PDMS from the back. Given that only a small portion of the filamentous cell apex extends in the PDMS, we removed the contribution from friction/adhesion due to the displacement of a 1-mm-diameter probe within PDMS by subtracting the $F_{\text {out }}$ value from the
$F_{\text {invas }}$ (Fig. 6c). These corrected $F_{\text {invas }}$ values, i.e. $F_{\text {in }}$, were largely independent of probe displacement rates over a range equivalent to cell filament extension rates when scaled down $(0.2-0.4 \mu \mathrm{m} / \mathrm{min})$. We used the equation that was established for $S$. pombe by Minc and colleagues;

$$
\frac{V_{(F)}}{V_{o}}=\left(1-\frac{F_{(\mathrm{PDMS})}}{\pi R^{2} \Delta P}\right)
$$

$V_{(F)}$ and $V_{o}$ are the filament extension rates within PDMS and on the surface, respectively; $F_{\text {(PDMS) }}$ is the resistive force of PDMS during filament displacement within this material $\left(F_{\text {in }}\right) ; R$ is the filament radius; and $\Delta P$ is the effective turgor pressure. The $F_{(\mathrm{PDMS})}$ was $1.5 \pm 0.7 \mathrm{~N}$ and $0.7 \pm 0.3 \mathrm{~N}$ at PDMS to cross-linker 35:1 (Young's modulus of $150 \mathrm{kPa}$ ) and 40:1 (Young's modulus of $100 \mathrm{kPa}$ ), respectively; scaling to the size of the hyphal filament (radius $1.04 \mu \mathrm{m}$ for surface growth) yielded $6 \pm 3 \mu \mathrm{N}$ and $3.2 \pm 1.4 \mu \mathrm{N}$, respectively. From these 


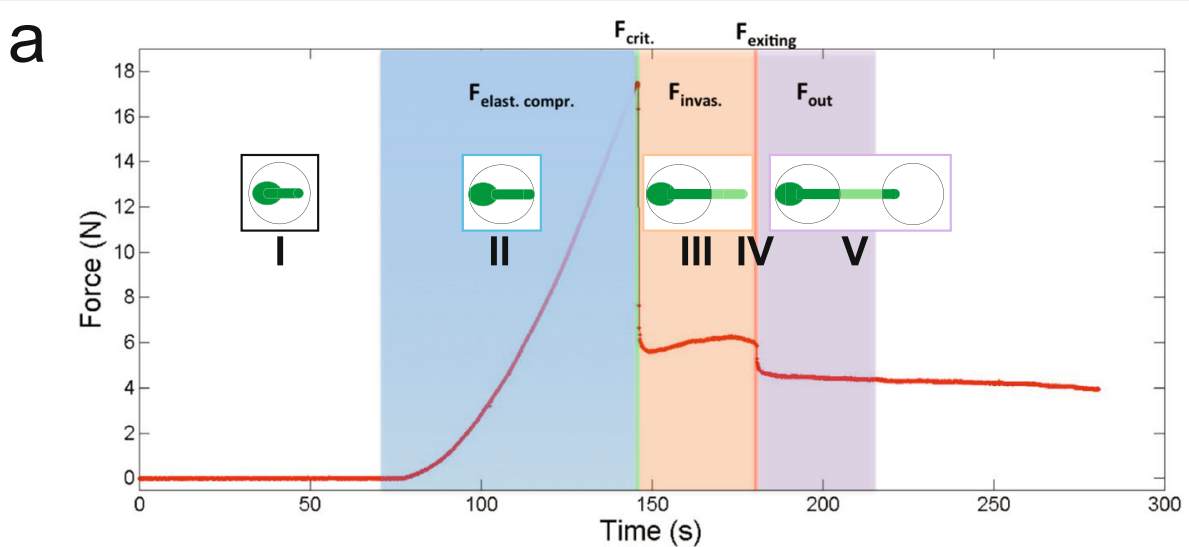

b

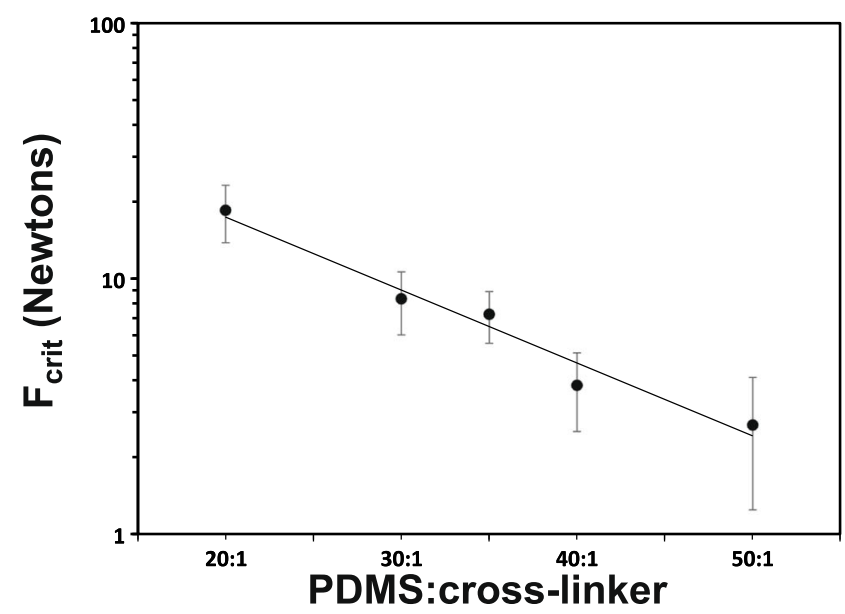

C

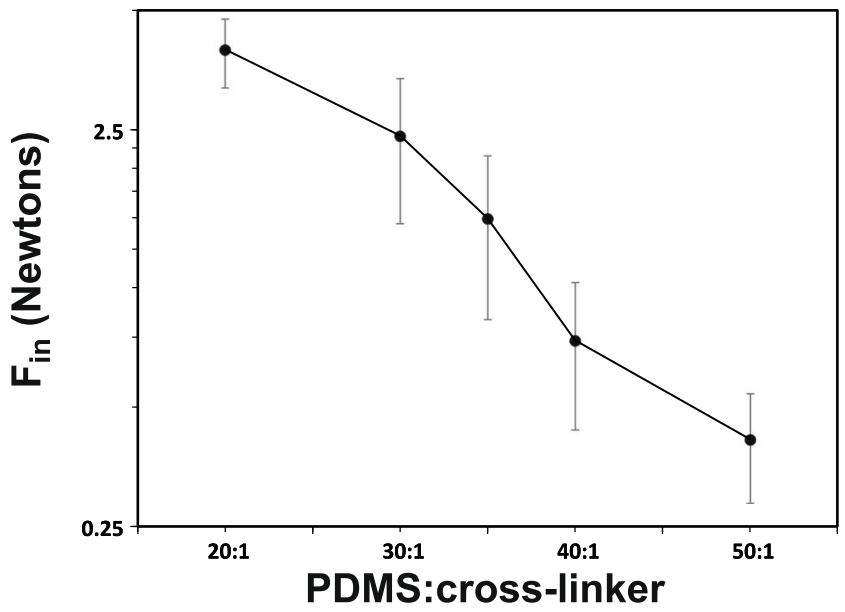

Fig. 6 Forces in physical model of PDMS invasive growth. a Forces encountered upon probe displacement into and out of PDMS. A 1-mmdiameter steel probe was advanced perpendicularly into the base of the cylindrical piece of cured PDMS (10:1 PDMS to cross-linker ratio, Young's modulus $\sim 2 \mathrm{MPa}$ ). Probe displacement rate was $63 \mu \mathrm{m} / \mathrm{s}$, and force was determined during different stages, as indicated, i.e., compression, penetration, and after the probe tip emerged from PDMS. Analogous stage of hyphal filament invasive growth indicated in insets along with the growth stage from time-lapse in Fig. 3a (I-V). b, c Resistive forces as a function of PDMS to cross-linker ratio. $\mathbf{b} F_{\text {crit }}$ was determined using a 1mm-diameter steel probe, with either 3.5- or 8-cm-diameter PDMS cylinders of indicated PDMS to cross-linker ratio, with probe displacement set to $1.6-3.2 \mu \mathrm{m} / \mathrm{s}$. Bars are SD for on average 10 independent determinations for each PDMS ratio. $\mathbf{c} F_{\text {in }}$ was determined by subtracting $F_{\text {out }}$ from $F_{\text {invas }}$ from the probe penetration experiment described in $\mathbf{a}$. Bars are SD 


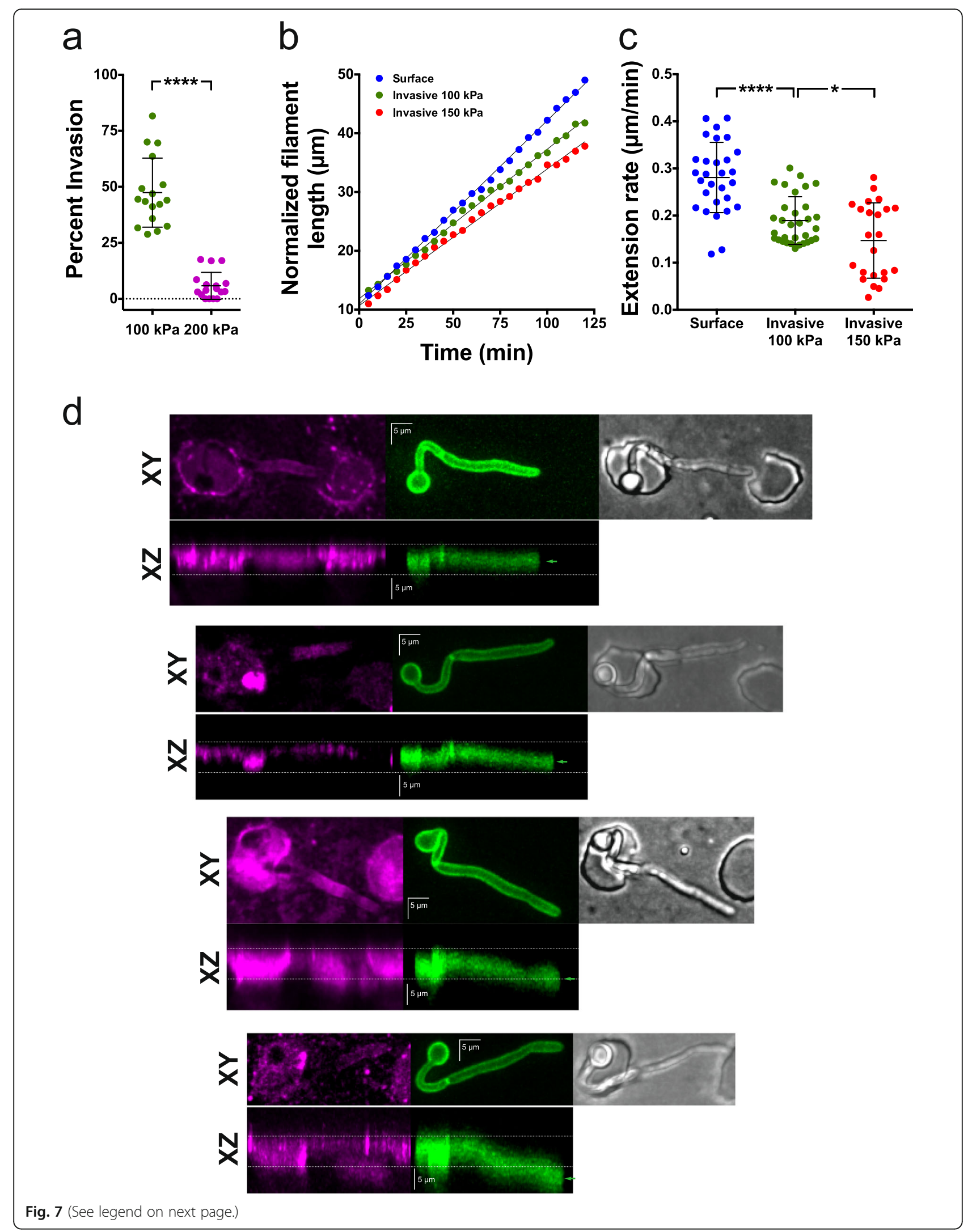




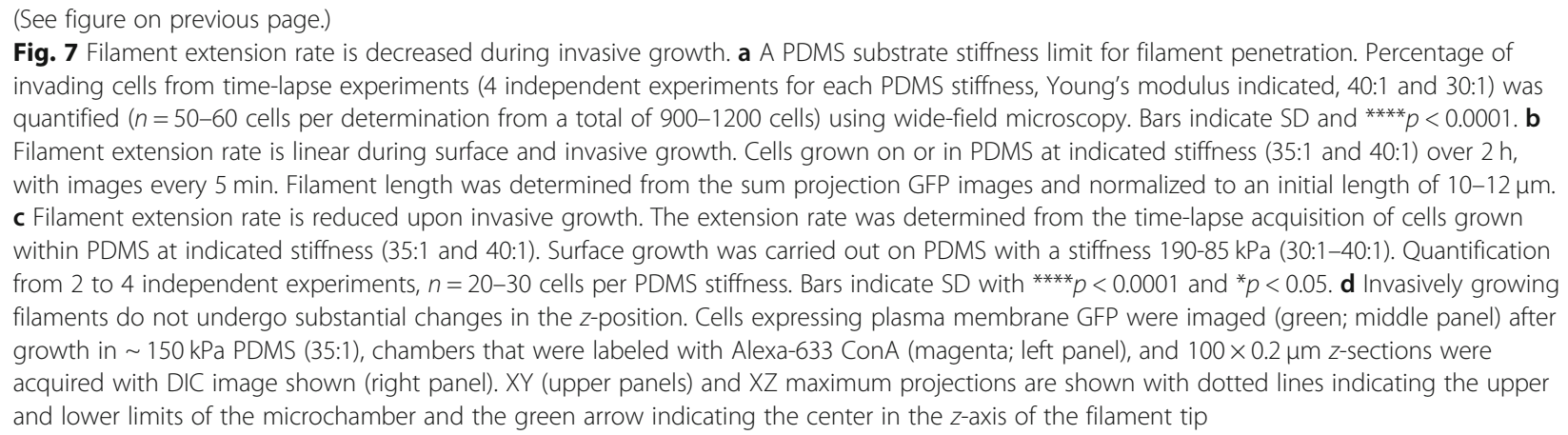

values, we determined the effective turgor pressure, $\Delta P$, to be $6 \pm 3 \mathrm{MPa}$ and $2 \pm 1 \mathrm{MPa}$ in these two conditions, respectively. Given that the hyphal filament diameter increases during invasion (radius $1.42 \mu \mathrm{m}$ at Young's modulus of $150 \mathrm{kPa}$ and $1.24 \mu \mathrm{m}$ at Young's modulus of $100 \mathrm{kPa}$ ), the calculated $\Delta P$ are $3.1 \pm 1.5 \mathrm{MPa}$ and $1.5 \pm$ $0.7 \mathrm{MPa}$, suggesting that the hyphal turgor pressure is 1-3 MPa. This value for turgor pressure is within the range reported both for planktonic and biofilm C. albicans cells, $\sim 1.2 \mathrm{MPa}[19]$ and $\sim 2 \mathrm{MPa}$ [34], respectively, as well as $S$. pombe, $0.85-1.5 \mathrm{MPa}[21,35]$. Nonetheless, it must be noted that these values are effective turgor pressure. In other words, $\Delta P$ is the turgor pressure exceeding the critical stress needed to deform the cell wall [21]. Hence, a combination of local compartment turgor pressure alteration, difference in cell wall deformability, or potentially finer adjustments in tip geometry may play important roles in penetration and invasion.

\section{Resistive force affects cell polarity}

The change in morphology during invasion, resulting in shorter and wider cells, could be explained by tip growth becoming more isotropic in response to a resistive force, raising the possibility that cell polarity is adversely affected. In $S$. pombe, it was observed that reducing the growth rate chemically, genetically, or mechanically destabilized active Cdc 42 polarization, a cell polarity master regulator [36]. To investigate whether cell polarity was altered in hyphal filaments growing invasively in PDMS, we examined the distribution of active Cdc42 (Cdc42•GTP), using a CRIB-GFP reporter [37]. Surprisingly, we observed a striking increase in polarized active Cdc42 at the filament tip throughout invasive growth, compared to surface growth (Fig. 11a, b). We determined, using a tailor-made MATLAB program, that this results from an increase in the concentration of Cdc42.GTP at the tip, rather than an alteration in the position of the maximum signal or spread of active Cdc42 further down the filament (Additional file 1: Figure S5A-D). These results suggest that, in response to a resistive force, there is an increase in cell polarization, perhaps reflecting a direct response to such external forces. We speculate that this higher level of active Cdc42 during invasive growth is due to the increased recruitment of the Cdc42 activator, Cdc24 [38]. We next examined active Rho1, as cell wall stress mediated by the cell surface mechanosensors Wsc1/Mid2 results in Rho1 depolarization in S. cerevisiae $[39,40]$. Figure $11 \mathrm{c}$ and d show that, in contrast to the increase in tip localized active Cdc42, active Rho1 is depolarized during invasive growth. We attribute this depolarization of active Rho1 to the mechanical properties of PDMS, which are likely to impose a uniform force over the hyphal filament surface, in addition to the resistive force in response to the tip extension.

The increase in tip-localized active Cdc42 during invasive growth suggests that the increase in filament diameter does not result from growth becoming more isotropic in response to resistive force. To examine whether this morphological change results from mechanical forces, we compared cell morphology over time during growth on the surface and within PDMS. Figure $12 \mathrm{a}$ and $\mathrm{b}$ show that the relative filament diameter (D1) was not altered during surface growth (mean diameter $2.26 \pm 0.15 \mu \mathrm{m}$ initially compared to $2.44 \pm 0.06 \mu \mathrm{m}$ after $2 \mathrm{~h}$ growth), in contrast to the invasive growth where there was a striking increase $(2.38 \pm 0.22 \mu \mathrm{m}$ initially compared to $2.98 \pm 0.15 \mu \mathrm{m}, p<0.0001)$. Specifically, the diameter of the filament compartment increased even $>10 \mu \mathrm{m}$ back from the tip (Fig. 12b). This $\sim 25 \%$ increase in diameter during invasive growth could either occur upon tip growth or subsequent to tip growth. The hyphal tip diameter was constant over $2 \mathrm{~h}$ of invasive growth and only slightly wider than that of cells growing on the surface $(2.53 \pm 0.11 \mu \mathrm{m}$ compared to $2.24 \pm$ $0.09 \mu \mathrm{m} ; p=0.0003$ ) (Fig. 12c, d). In contrast, Fig. 12d shows that there was a significant difference between the mean diameter at the tip of the apical cell and that of the cell proximal to the apical cell during invasive growth (mean proximal cell diameter $2.99 \pm 0.17 \mu \mathrm{m} ; p<$ 0.0001). Together, these results indicate that relatively small changes in the tip morphology are not sufficient to 

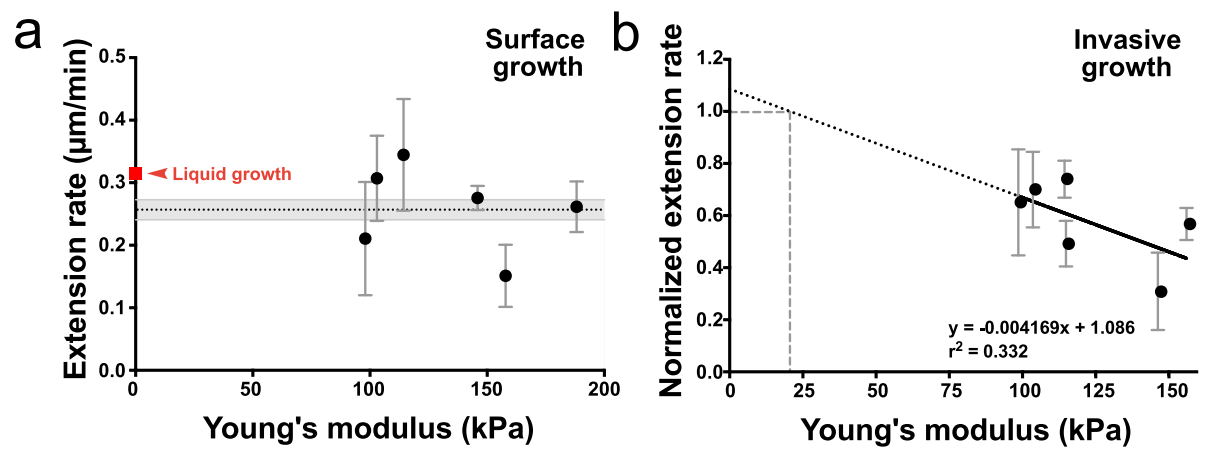

Fig. 8 Filament extension rate on the PDMS surface is slightly reduced compared to that in liquid media. a Filament extension rate on the PDMS surface is independent of stiffness and reduced compared to that in liquid media. The extension rate was determined as in Fig. 7c. Filament lengths of cells grown in serum-containing liquid media ( $n=75-80$ cells) were measured every 30 min with mean and SEM shown in red. Surface extension rates determined as in Fig. 7c ( $n=30$ cells; 3-9 experiments per PDMS stiffness). Bars indicate SD. The mean of all surface extension rates (dotted black line) and SEM (gray zone) are indicated. b Cells experience a resistive force on the PDMS surface. Invasive extension rates determined as in Fig. 7c ( $n=53$ cells; 5-10 experiments per PDMS stiffness) and normalized to surface extension rates, with bars indicating SD and dotted line the best fit. At $Y=1, X$ is $\sim 20 \mathrm{kPa}$

explain the altered morphology of the filament, back from the tip, which are due to external mechanical forces.

\section{Discussion}

We used PDMS micro-fabrication to probe the relationship between substrate stiffness and growth of $C$. albicans filamentous cells. Below a stiffness threshold of $\sim 200 \mathrm{kPa}, C$. albicans can penetrate and grow within PDMS. The chemical inertness of this polymer, as well as the observed well deformation, suggest that turgor pressure-driven active penetration is critical for this invasive growth. C. albicans filamentous growth within a stiff substrate is characterized by dramatic filament buckling, which correlates with the position of cell division sites, in addition to a stiffnessdependent decrease in extension rate. Growth within

\section{a}
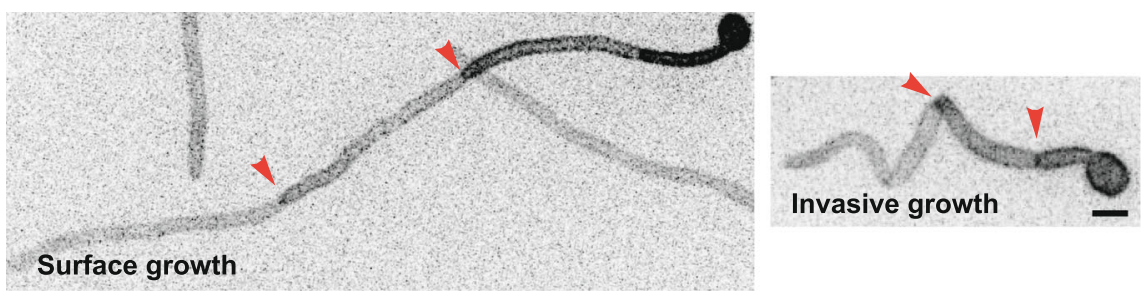

b
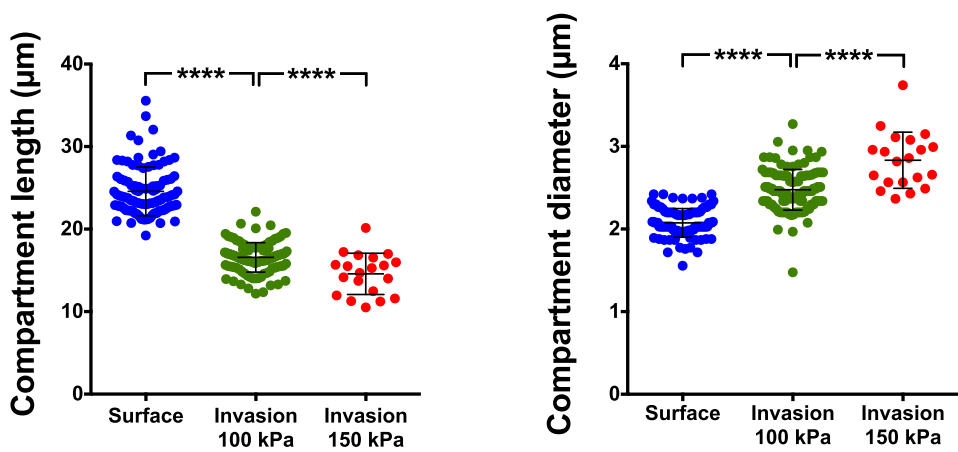

Fig. 9 Filament morphology is altered during invasive growth. a Sum projection images of cells expressing GFP-Ct $t_{\text {Rac1 }}$ grown in the presence of FCS on or within PDMS of $115 \mathrm{kPa}$ stiffness, for $3 \mathrm{~h} 15$ or $2 \mathrm{~h} 15$, respectively. Arrowheads indicate the septa that delimit the measured compartment. $\mathbf{b}$ Compartment length is reduced and diameter is increased when cells are grown within a substrate of increasing stiffness (40:1 and 35:1). The length between two septa (left panel) along with the filament diameter (right panel), either the 2nd or the 3rd compartment from the tip, was measured in $n=20-115$ cells, 2-22 experiments. SD are indicated and $p<0.0001$ 
a

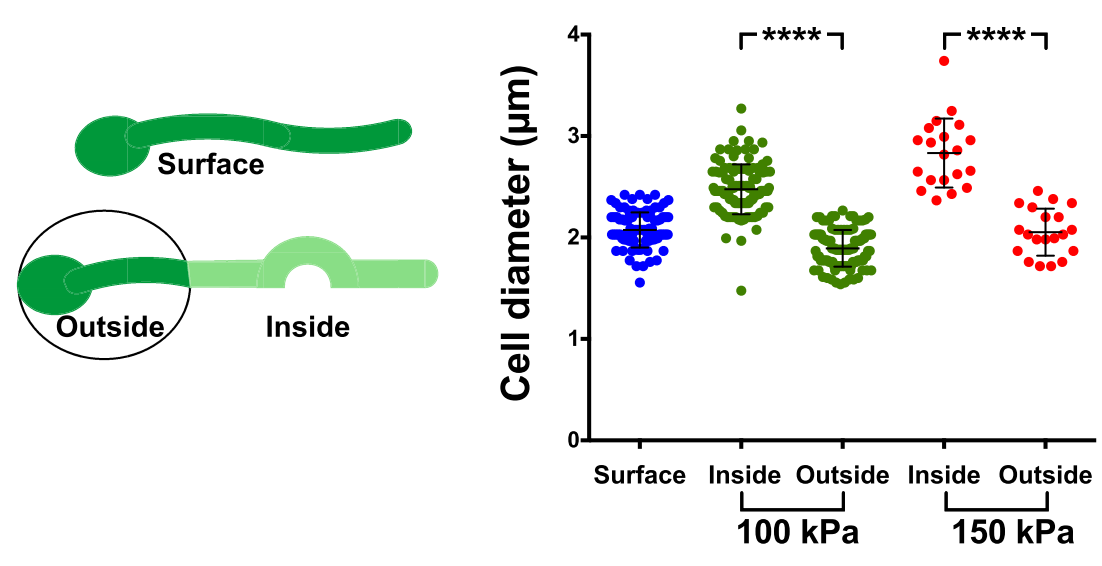

b

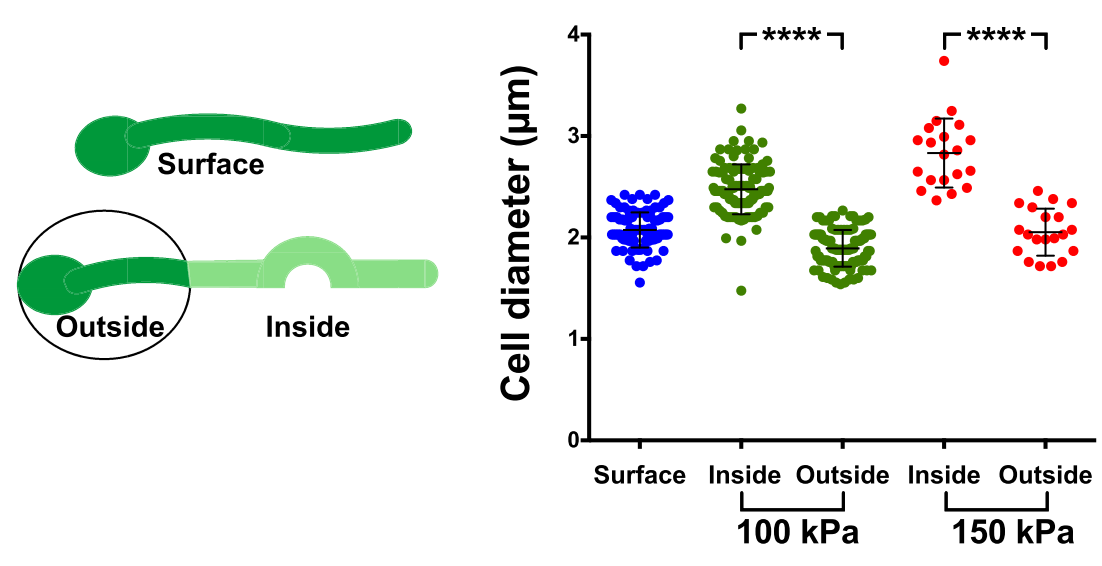

$90 \mathrm{kPa}$
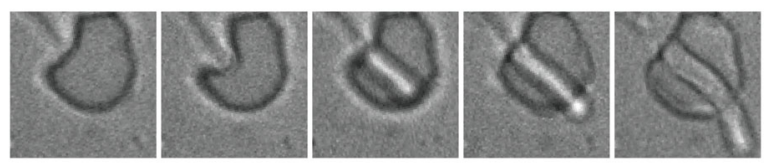

$250 \mathrm{kPa}$
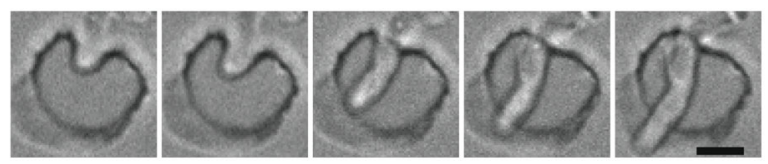

C
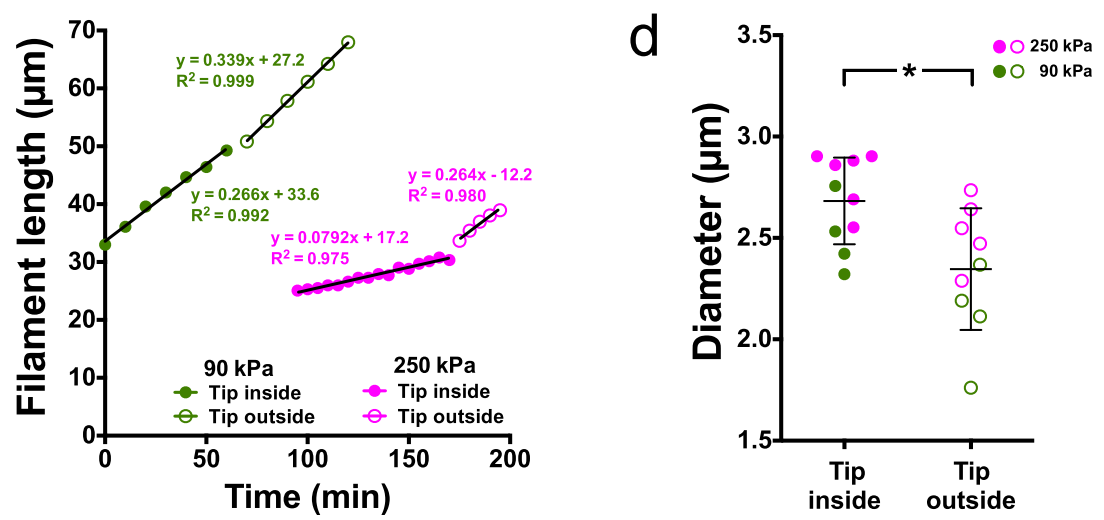

Fig. 10 Filament diameter increases during growth in PDMS and decreases upon bursting out. a Filament diameter inside and outside PDMS at two stiffness values. The diameter of the filament was measured as in Fig. 9a and b (surface and portion inside PDMS). The diameter of the filament adjacent to the mother cell neck in the chamber was measured (portion outside indicated in the schematic). Bars indicate SD, with $p<$ 0.0001 between inside and outside for each PDMS stiffness value (40:1 and 35:1). b Images of filament bursting out of PDMS. Cells grown in PDMS at indicated times over $2 \mathrm{~h}$, with DIC images every 5 min ( $250 \mathrm{kPa} ; 30: 1)$ or 10 min $(90 \mathrm{kPa} ; 40: 1)$ shown. c Filament extension rate increases upon exiting PDMS. Filament length was measured from the sum projection GFP images prior to (green and magenta-filled symbols) and following emerging from PDMS (green and magenta-open symbols). $\mathbf{d}$ Filament tip diameter is slightly reduced upon emerging from PDMS. The tip diameter was determined at $4-5$ times before or after exiting PDMS from $\mathbf{b}$ and $\mathbf{c}$. Bars indicate SD and ${ }^{*} p=0.01$

a substrate also resulted in a striking alteration in morphology, i.e. reduced cell compartment length and increased diameter. Our results reveal that changes in morphology are not due to a depolarization of active Cdc42, but rather to the mechanical forces from the substrate.

\section{Growth behavior as a function of substrate stiffness}

Substrate stiffness determines whether C. albicans will grow on a surface or within it, below a threshold corresponding to a stiffness of $200 \mathrm{kPa}$. During growth within PDMS, a substantial fraction of filaments buckle, with greater than $50 \%$ buckling in a substrate with a Young's modulus of $\sim 100 \mathrm{kPa}$. Cells trapped in stiff (200 kPa or greater) microchambers undergo dramatic subapical bending. While both filament buckling and subapical bending depend on filament extension, the former occurs at least $5-10 \mu \mathrm{m}$ away from the apex whereas the latter occurs at the filament tip. In a number of cases, filaments buckled as the tip (within PDMS) 

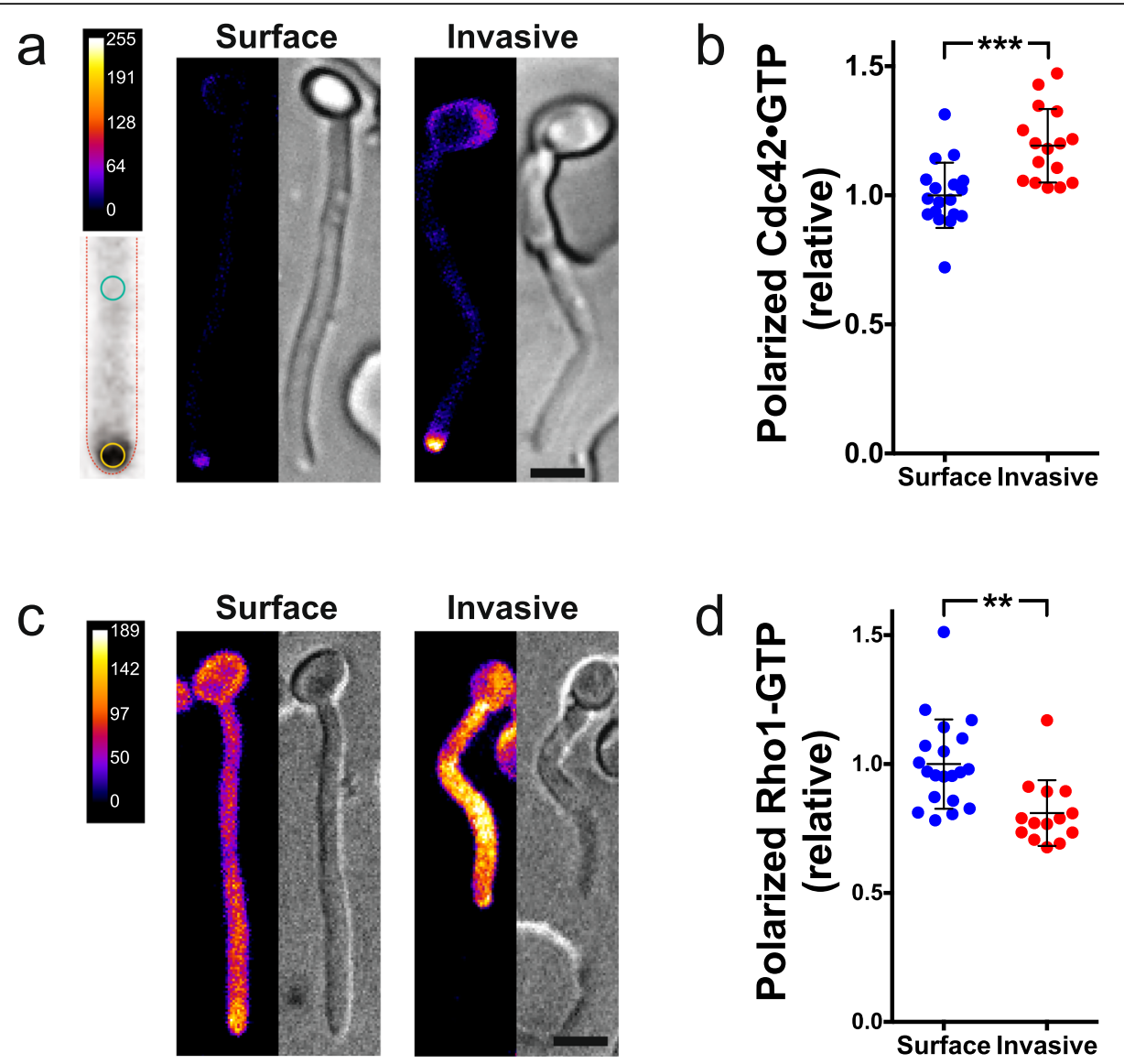

Fig. 11 Invasively growing filaments have increased levels of active Cdc42 at the tip. a, b Tip-localized Cdc42.GTP is increased during invasive growth. a Representative sum projection and DIC images of cells expressing CRIB-GFP on or in 35:1 PDMS ( 150 kPa). False colored sum projection of $23 \times 0.4 \mu \mathrm{m} z$-sections (LUT, top) with schematic indicating regions quantitated at the tip and $5-10 \mu \mathrm{m}$ back from the tip (bottom). b Mean Cdc42.GTP at the tip and 5-10 $\mathrm{mm}$ subapically determined from 4 independent time-lapse experiments (images every $5 \mathrm{~min}$ for $\sim 2 \mathrm{~h}$ and sum projections of $23 \times 0.4 \mu \mathrm{m} z$-sections; $n=16-18$ cells). Polarized Cdc42.GTP is the tip signal divided by the subapical signal, $5-10 \mu m$ behind the tip (3.5-fold enrichment apically for surface growing cells, normalized to 1). Bars indicate SD and *** $p=0.0002$. $\mathbf{c}, \mathbf{d}$ Active Rho1 is delocalized during invasive growth. $\mathbf{c}$ Representative sum projections and DIC images as in a of cells expressing GFP-RID after growth on or in 35:1 PDMS ( $150 \mathrm{kPa})$. d Mean Rho1·GTP at the tip and at 5-10 $\mu \mathrm{m}$ subapically was determined from 3 independent time-lapse experiments, as in $\mathbf{b}(n=14-20$ cells). Polarized Rho1·GTP is the tip signal divided by the subapical signal (1.4-fold enrichment apically for surface growing cells, normalized to 1 , as in $\mathbf{b}$ ). Bars indicate SD and ${ }^{* *} p=0.001$

approached a well, followed by partial release upon penetration into this well. This bursting out event is similar, in some respects, to damaging of or escaping from host cells, such as macrophages [28-31].

Despite the estimated resistive force during invasive growth of 3-10 $\mu \mathrm{N}$ at the hyphal scale, we did not observe dramatic changes in the filament tip shape. Interestingly, the location of cell division sites appeared to be affected by filament buckling, in that there was a high correlation between the division site location and the site of buckling. Septins have been shown to be enriched preferentially at locations of high curvature in Ashbya gossypii hyphal filaments, i.e. at branch points [41]. An attractive possibility is that the location of the cell division site at the buckle, or vice versa, may minimize physical stress or damage to the filament.
Effects of substrate stiffness on invasive growth

Filament extension rate is reduced, and cell compartment length decreases with increasing substrate stiffness; however, cell volume is largely unaffected during growth within $100 \mathrm{kPa}$ PDMS, due to an increase in filament diameter. These results suggest that the overall growth rate is unaffected in this condition, raising the possibility that the cell tip growth area is altered by resistive force. Given that altering growth in S. pombe by chemical, genetic, or mechanical means, results in destabilization of the active Cdc42 cluster at the growth site, we investigated the distribution of this key GTPase during invasive growth. Surprisingly, the distribution of active Cdc42 was not altered during invasive growth, but rather there was an increase in the concentration of active Cdc42 at the tip. In contrast, active Rho1, which is critical for glucan synthesis, was less polarized and 
a

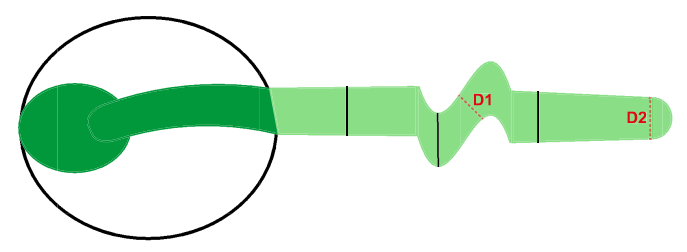

b

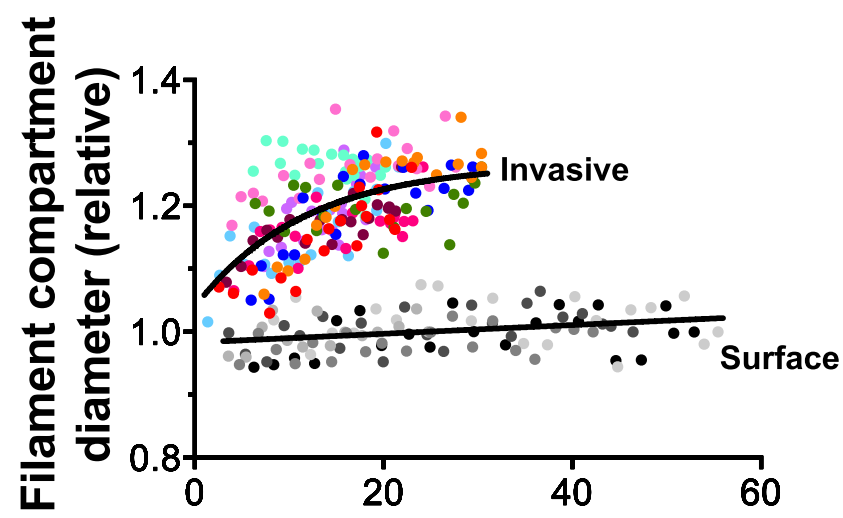

\section{Distance from compartment to tip $(\mu \mathrm{m})$}

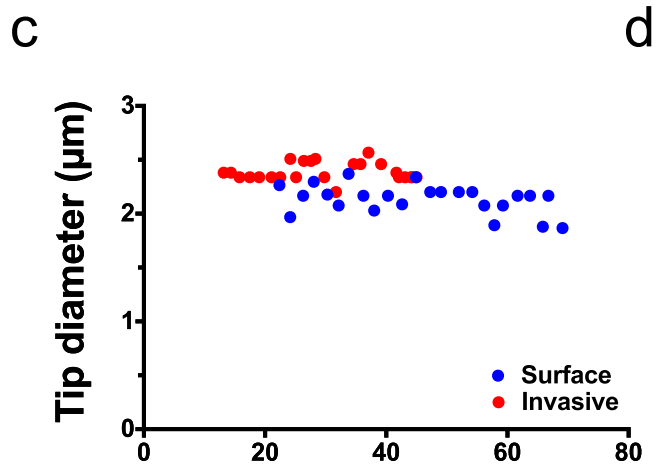

Filament length $(\mu \mathrm{m})$

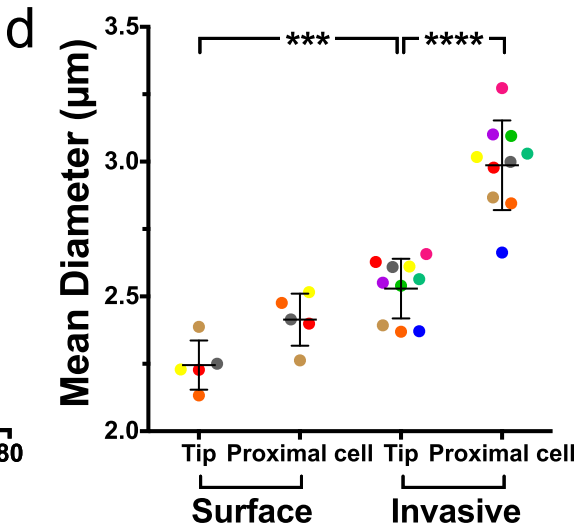

Fig. 12 Mechanical forces are critical for filament morphology changes. a Schematic of filament during invasive growth. Lighter green indicates a portion of the filament within PDMS; D1, compartment or proximal cell diameter; and D2, tip diameter. $\mathbf{b}$ The diameter of the cell compartment increases as it becomes further away from the filament tip during invasive growth. Cell compartment diameter (measured at 5 equidistant positions between 2 septa), initially on average $5 \mu \mathrm{m}$ from tip, was determined from 3 independent experiments acquired as in Fig. 11b ( $n=5-10$ cells) grown on or within 35:1 PDMS ( 150 kPa). Distance from the compartment center to the tip was determined at each time. The diameter was normalized to the diameter of the last time point for each cell and further normalized by the mean difference between the final compartment diameters of invasive cells compared to surface cells. Each color represents a cell. c Filament tip diameter does not vary as a function of filament length for surface and invasive growth. Tip diameter $(2 \mu \mathrm{m}$ back from the apex) measured for one cell growing on PDMS surface and one cell growing within PDMS, over a 2-h time course. $\mathbf{d}$ Tip diameter increase does not underlie proximal cell diameter increase during invasive growth. Tip and proximal cell diameters were measured from the same cells as in a. Proximal cell diameter was measured at the center of the cell proximal to the apical cell over the 2 nd hour of the time course (filaments $>30 \mu \mathrm{m}$ long). Bars indicate $S D, w_{i t h}{ }^{* * *} p<0.0001$ between the tip and the proximal cell during invasive growth and ${ }^{* *} p=0.0003$ between the invasive and surface tips

observed throughout the filament during invasive growth. Together, these results suggest that, during invasive growth, cells sense resistive force from the PDMS and polarization increases at the tip, while remodeling their cell walls throughout the filament to counter this resistive force and/ or the increase in turgor pressure. A likely possibility is that this resistive force is sensed by the mechano-sensing pathway, via a module composed of the mucin Msb2 and the cell wall protein Sho1 [42] critical for S. cerevisiae survival during compressive stress. In this yeast, Msb2 localizes to sites of growth and binds active Cdc42 [43], and cell wall stress has been shown to depolarize Rho1, as well as to 
hyperactivate this GTPase $[39,40]$ via the cell surface sensors, Wsc1 and Mid2. Indeed, S. cerevisiae cells sense compressive stress via Mid2, which localizes uniformly at the plasma membrane [44]. We speculate that the increased compression around the hyphal filament leads to depolarized active Rho1 via Mid2, in contrast to localized active Cdc42 via Msb2.

We have used a viscoplastic model for fungal growth that was originally established to describe cell shape control in plants $[45,46]$ and used, more recently, to analyze cell growth in fission yeast [21]. In such models, viscoplastic deformation of the cell wall underlies growth, which is driven by high turgor pressure. Specifically, the pressure $(P)$ has to exceed the threshold plastic yield strain $\left(P_{c}\right)$ of the cell wall-the cell growth rate is proportional to the wall strain that exceeds this threshold: $V_{o} \propto \frac{\left(P-P_{c}\right)}{E_{\mathrm{CW}}}$. Hence, we have used Eq. (1) to derive the effective turgor pressure in C. albicans hyphae. Here, we used the differences in growth rates on PDMS surface and within PDMS, as well as an approximation of the external force. This latter force was calculated based on the displacement within PDMS of a 1-mm-diameter steel probe, minus the contribution from friction/adhesion and scaled to the hyphal diameter, assuming scaling with respect to the cross-sectional area and the absence of dramatic changes in geometry. From this analysis, we determined the effective turgor pressure $\left(\triangle P=P-P_{c}\right)$ to be 1.5 and $3.1 \mathrm{MPa}$, in $100 \mathrm{kPa}$ and $150 \mathrm{kPa}$ PDMS, respectively. Either increasing filament diameter or decreasing extension rate results in a lower $\Delta P$. The physical experimental model, however, does not take into account the geometry of the hyphal tip, which has a different curvature than the metal probe, which could result in an overestimation of the forces in the physical model. More precise measurements of forces relevant to hyphae will require a more accurate description of the hyphal tip size and geometry. One possibility is that, upon growth in stiffer PDMS, there is an increase in turgor pressure; our results are consistent with such a scenario as $\Delta P$ increases from 1.5 to $3.1 \mathrm{MPa}$, with an increase in PDMS stiffness from 100 to $150 \mathrm{kPa}$. This increase in turgor pressure could be responsible for the increased volume of the invasive cell compartment within $150 \mathrm{kPa}$ PDMS $\left(92 \mu \mathrm{m}^{3}\right.$ compared to $83 \mu \mathrm{m}^{3}$ for surface growing cells, $p=0.045$ ). Previous studies on cell wall expansion in S. pombe [35] have shown that $\frac{\Delta P}{Y}=\frac{\left(R^{*}\right) t}{R_{1}}$; where $Y$ is the cell wall Young's modulus; $R^{*}$ is the expansion ratio in width $\left(\left[R_{1}-R_{0}\right] / R_{0}\right) ; R_{1}$ and $R_{0}$ are the cell radius within the PDMS and on the surface, respectively; and $t$ is the cell wall thickness $(\sim 0.2 \mu \mathrm{m})$. Assuming $Y$ is constant at these two PDMS stiffness conditions, $\frac{\Delta P}{Y}(35: 1)$ is 1.7 -fold greater than $\frac{\Delta P}{Y}(40: 1)$, consistent with the observed increase in turgor pressure we derived from the growth rate difference. The slightly reduced extension rate on PDMS surface compared to that in liquid, together with the extrapolation of normalized extension rate as a function of PDMS stiffness to where $\frac{V_{(F)}}{V_{o}}=1$, indicates that during growth on a surface, the hyphae experience a small resistive force, equivalent to growth within less stiff PDMS $(\sim 20 \mathrm{kPa})$, which we attribute to adherence/friction. It is likely that depending on the cellular surface, the contribution of adherence/friction will vary.

\section{Cell morphology is dependent of substrate stiffness}

Our results indicate that effects on cell morphology are only observed in filaments within the PDMS, suggesting that resistive forces from the PDMS lead to an alteration in morphology. Strikingly, during growth in the stiffest PDMS, we observed a progressive increase in compartment diameter, even when this compartment was more than $10 \mu \mathrm{m}$ back from the hyphal tip. This could be due to the additional modification of the cell wall in this proximal compartment, i.e. resulting in a less stiff cell wall, an increase in turgor pressure, and/or mechanical deformation of the filament. Although we cannot rule out that the cell wall in this proximal compartment is less stiff during invasive growth, we favor the latter two possibilities, as the cell compartment volume increases and $\sim 60 \%$ of invasively growing hyphae buckle in this PDMS stiffness $(150 \mathrm{kPa})$. The dramatic alteration in filament morphology was not solely due to a widened tip, as it only increased slightly during invasive growth, compared to the proximal compartment that increased $25 \%$ more than the tip diameter. It is likely that these morphology changes are due to the mechanical forces from growing against a resistive substrate together with an increase in turgor pressure.

A number of fungal pathogens penetrate host tissues, including medically relevant $C$. albicans $[8,10,12]$ and A. fumigatus [9], and plant pathogens [47, 48], including Colletotrichum sp. [49], Ustilago maydis [50], Magnaporthe sp. [51, 52], and Fusarium sp. [53]. For the latter plant pathogens, high turgor pressure is generated inside a specialized cell, called an appressorium, that generates pressures in excess of $8 \mathrm{MPa}$ [51]. Blocking host cell endocytosis of the human fungal pathogens $C$. albicans [8, 15-17] and A. fumigatus [9] with Cytochalasin D has revealed that both of these fungi can enter epithelial tissue by active penetration. Young's moduli for mammalian host cells are in the $1-100 \mathrm{kPa}$ range. Hence, the effects we observe on filament extension rate and morphology are likely to be relevant during active penetration of epithelial cells, and it is attractive to speculate that turgor pressure, in particular the osmolytes critical 
for generating this force, might be a target for antifungal drugs.

\section{Conclusions}

Our results suggest that the stiffness of host cells dictates which cell type $C$. albicans can penetrate. Interestingly, even with stiffer PDMS ( $200 \mathrm{kPa})$, we observed a small percentage of cells that were able to invade the substrate, suggesting these cells have specific properties that could be an advantage during epithelium invasion.

\section{Methods}

\section{Strains, media, and genetic methods}

Standard methods were used for C. albicans cell culture, molecular, and genetic manipulations as described. Derivatives of the BWP17 strain were used in this study and are listed in Table S1. Strains were grown in rich media (yeast extract peptone dextrose) at $30^{\circ} \mathrm{C}$ for all experiments, and induction of filamentous growth was carried out with fetal calf serum (FCS) at $37^{\circ} \mathrm{C}$. Oligonucleotides and synthesized DNA used in this study are listed in Tables S2 and S3. pDUP5-mScarlet-Ct $\mathrm{C}_{\text {Rac1 }}$ was generated by PCR amplification of CamScarlet with a unique 5' AscI site and a 3' CtRac1 followed by a unique MluI site (oligonucleotides CamScarletAscIp and yemChmCtRacMluI) and cloned into pDUP5-ADH1p-

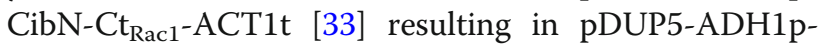
CamScarlet-Ct $t_{R a c 1}$-ACT1t. The nucleotide sequence for RFP mScarlet [54] was codon optimized for C. albicans and commercially synthesized (Genscript). mScarlet was PCR amplified with unique PstI and AscI sites (oligonucleotides GA3CamScarPstIp and CamScarletmAscI) and cloned into a pFA-GFPy-URA3 backbone [55] resulting in pFA-CamScarlet-URA3. The RID, CRIB, and $\mathrm{Ct}_{\text {Rac1 }}$ plasmids were linearized with StuI or NgoMIV and transformed into strains.

\section{Micro-fabrication}

Microchambers were fabricated using standard softlithography methods [56, 57]). Chambers were $5 \mu \mathrm{m}$ deep and $10 \mu \mathrm{m}$ in diameter with $15 \mu \mathrm{m}$ spacing between them. The overall thickness of microchamber preparations was approximately $150 \mu \mathrm{m}$. PDMS microchambers of varying stiffness were generated by varying the ratios of polymer and cross-linker (Sylgard 184; Dow Corning). Polymer-cross-linker mixtures were spin coated (Laurell Technologies corporation) on molds $(5 \mathrm{~s} \quad 100 \mathrm{rpm}$ followed by $50 \mathrm{~s} 500 \mathrm{rpm}$ ) to achieve $\sim 150 \mu \mathrm{m}$ thickness and then cured for $1 \mathrm{~h}$ at $60^{\circ} \mathrm{C}$ PDMS (10:1). Thick PDMS frames were then placed on top of the partially cured chambers, which were baked for an additional $2 \mathrm{~h}$ at $90^{\circ} \mathrm{C}$, in order to peel the thin chambers off the mold. For viscoanalyzer measurements, $20 \times 20 \times 5 \mathrm{~mm}$ PDMS pieces were similarly cured and samples subjected to an amplitude of oscillation of $5-150 \mu \mathrm{m}$ at $10 \mathrm{~Hz}$ frequency using a DMA3007000 Metravib 52 (Areva) with Dynaset20 software. Young's moduli were calculated using the following formula, $E=\frac{\sigma}{\varepsilon}=\frac{F \times h}{D \times l \times e}$; with $F$ being the force, $D$ the deformation amplitude, $H$ the sample height, $e$ the sample thickness, and $l$ the sample length. For analysis of each PDMS preparation, measurements at 5 different deformation values $(5-150 \mu \mathrm{m})$ were carried out, and the mean of 3 of these measurements was used for Young's modulus determination. For indentation experiments, varying ratios of polymer and crosslinker were poured into two different sized cylindrical aluminum molds, $3.5(d) \times 1.75 \mathrm{~cm}(h)$ and $8(d) \times 2 \mathrm{~cm}$ $(h)$, and cured as described above.

\section{Physical model of penetration}

Half of the PDMS cylinders were adhered to an aluminum plate with a 5-mm-diameter hole. For the other half of the PDMS cylinders, the bottom of the mold was removed such that they were fixed around their circumference. Cylindrical PDMS pieces from both conditions were indented in their center with a hemispherical 1-mm-diameter steel probe, which was highly polished. This probe tip was machined with a slight conical shape ending in a spherical cap to approximate a hyphal filament tip and had a 2-daN force sensor (Kistler), as well as a displacement sensor (Fastar Data Instruments) attached. This probe was extended using a moving deck with a motor (Cerclet) at speeds of 1.6-3.2 $\mu \mathrm{m} /$ s. Measurements were made on average 10 samples at each PDMS ratio. From force versus displacement curves, the following forces were derived: the maximum value was the $F_{\text {crit }}$, the mean of the first plateau following penetration $F_{\text {invas }}$, and the mean of the second plateau (following the exit of the PDMS) $F_{\text {out }}$. Forces were then averaged and scaled to the cross-sectional area of the hyphal filament. We assumed that in the physical model of penetration and in the C. albicans growth experiments, the PDMS behaved as a homogenous material, and hence, scaling in terms of size was carried out with a constant critical stress. This assumption is justified, as the diameter of the filament, $\sim 2 \mu \mathrm{m}$, is $10^{3}$-fold greater than the PDMS mesh size, which is $2-3 \mathrm{~nm}$ with 40:1 PDMS. Hence, scaling of the force, as stress during rupture is constant, was achieved by dividing for the ratio of the metal probe/filament radii squared.

\section{Microscopy sample preparation}

PDMS microchambers were activated by plasma treatment (Harrick Plasma Cleaner) for $14 \mathrm{~s}$ at 500 mTorr on low setting submerged in $\mathrm{DH}_{2} \mathrm{O}$ until usage. Prior to usage, PDMS samples were dried with nitrogen gas, treated with poly-D-lysine $(1 \mathrm{mg} / \mathrm{ml})$ and subsequently 
treated with concanavalin A $(0.4 \mathrm{mg} / \mathrm{ml})$, each incubated for 20 min followed by drying with a stream of nitrogen gas. Exponentially growing cells were mixed with FCS media (75\% FCS, $0.6 \times$ minimal media and $2 \%$ dextrose) and spotted onto the microchambers and were sealed with a coverslip. Typically, cells on PDMS microchambers were incubated for $\sim 1 \mathrm{~h}$ at $37^{\circ} \mathrm{C}$ prior to microscopy to initiate filamentation.

\section{Microscopy and image analysis}

Cells were imaged as described [33] using either spinning disk confocal microscopy or wide-field fluorescence microscopy with a UPLANAPO $1.2 \mathrm{NA} \times 60$ or PlanNeofluar $0.75 \mathrm{NA} \times 40$ objectives, respectively. Images were acquired at indicated times, with 0.4 or $0.5 \mu \mathrm{m} z$ sections (13 to 24) to capture the entire filament. For growth depth experiments, $100 \times 0.2 \mu \mathrm{m} z$-sections were acquired. For the analyses of extension rate, compartment morphology, tip morphology, and cell depth images were deconvolved with the Huygens Professional software version 18.04 (Scientific-Volume Imaging) with recommended settings and a signal to noise ratio of 10 , and sum projections were used. Reflection images in XZ were acquired on an upright Leica DM5500 TCS SPE laser-scanning microscope (Leica Microsystems, Mannheim, Germany) equipped with a galvanometric stage, using an APO 0.3 NA $\times 10$ objective. Reflection images were acquired in XZ reflection mode using a 488-nm laser and an 80/20 dichroic filter. Scale bars, unless otherwise indicated, are $5 \mu \mathrm{m}$.

Image analysis for extension rate, compartment/tip diameter, and active Rho GTPase polarization was carried out with Fiji (version 1.51) [58]. For the determination of the extension rate, the filament lengths (mother cell neck to filament tip) were determined over time using plasma membrane fluorescence signal and values are from a curve fit of minimally $1 \mathrm{~h}$ acquisition $(>12$ time points). For liquid extension rates, cells induced with $50 \%$ FCS at $37^{\circ} \mathrm{C}$ were fixed every $30 \mathrm{~min}$ and average cell lengths for each time point $(0-90 \mathrm{~min})$ were used. Compartment lengths were measured using the edge-to-edge fluorescence signal between two formed septa. Only invasive compartments that were entirely within the PDMS were used for analyses. Active Cdc42 and Rho1 polarization was determined using a fixed size ROI to quantitate the signal intensity at the tip and in a subapical region. The ratio of the tip to subapical signal was averaged over the time-lapse, and the mean for invasive cells was normalized to the mean ratio for noninvasive cells. The percentage of cells undergoing subapical bending within a chamber and filament buckling within PDMS was assessed using DIC images. For the determination of errors from values derived from multiple measurements, classical error propagation was carried out [59]. Statistical significance was determined with Student's $t$ test.

For the representation of 3D DIC images, an algorithm was applied to image $z$-stacks using a custom-developed MATLAB program called InFocus inspired from Extended Depth of Field plugin [60]. The program extracts from any 3D multi-channel acquisition, the local contrast per channel. From these maps, it determines the $Z$ of highest contrast per pixel from one or a combination of channels and then extracts a smoothened plane to get a 2D multichannel image. Parameters can be adapted through the graphical user interface. For quantitation of filament tip curvature over time, we developed another MATLAB program called TipCurve with an intuitive interface dedicated for morphological analyses along the major axis of filamentous cells. For such analyses, 3D images were converted into $2 \mathrm{D}$ images by sum projection, and similar to the HyphalPolarity program [33], a backbone was extracted from images over time, but also the cell contour, specifically extracting the tip first, we estimate the extremity of the backbone subtracted by the tip radius. Then, we estimate the local curvature only along the tip (either $-45^{\circ}$ to $+45^{\circ}$ or -90 to $90^{\circ}$ ) from the curvilinear function $\mathrm{f}(\mathrm{s})$ extracted from cell contour:

$$
C(s)=\frac{1}{R(s)}=\frac{f^{\prime \prime}(s)}{\left(f^{\prime}(s)\right)^{2}}
$$

The distribution of active Cdc42 and Rho1 at the filament tip was determined by TipCurve. For these analyses, images that had a polarized fluorescent signal along with a uniform cytoplasmic signal were used. The latter was used to identify the cell and extract the backbone. Then, an additive projection on the backbone was done to get kymograph curves per time point and per fluorescence channel. To estimate the distribution of intensity at the tip of Cdc42 and Rho1, we fitted the kymograph to estimate $x_{\max }$, the distance of maximum of intensity from the tip and the decay of intensity above $x_{\max }$ as an exponential function. Both Infocus and TipCurve can be provided as executables on demand.

\section{Supplementary information}

Supplementary information accompanies this paper at https://doi.org/10 1186/s12915-020-00833-0.

Additional file 1: Figure S1. Strain versus stress dependence of PDMS. Analyses carried out using a Viscoanalyzer, with oscillation at $10 \mathrm{~Hz}$ of PDMS at cross-linker ratio of 40:1. Figure S2. Invasive growth and penetration into adjacent chamber in PDMS of different stiffness. DIC timelapse experiments at indicated PDMS:cross-linker ratio and measured stiffness (Young's modulus). The adjacent chamber is highlighted with a dotted yellow line and deformation of this chamber lasted $\sim 40$ min with 40:1 PDMS ratio and 80-90 min for the two stiffer PDMS substrates. Figure S3. The shape of filament tip is not substantially altered during invasive growth in PDMS. A) Radius of curvature over time is constant in 
surface and invasively growing cells. Radius of curvature with an arc of \pm $90^{\circ}$ or $\pm 45^{\circ}$ at the filament tip. B) Shape of filament tip of surface growing cells over time. Cells were grown on PDMS (30:1; $250 \mathrm{kPa})$ and $31 \times 5$ min GFP sum projections were analyzed. Radius of curvature with $\pm 45^{\circ}$ by indicated open lines and $\pm 90^{\circ}$ indicated by solid lines. Figure $\mathbf{S 4}$. Cells confined within a stiff PDMS chamber have reduced filament extension rates. A) Constricted growth within a PDMS chamber. Typical timelapse experiment using $160 \mathrm{kPa}$ PDMS, with DIC images every 5 min shown. B) Filament extension rate within a stiff chamber is not linear. Filament length was determined from images every $5 \mathrm{~min}$ for $\sim 2 \mathrm{~h}$ and GFP sum projections ( $n=9$ cells). C) Filament extension rate is substantially reduced as chamber fills up. Initial (filament length 10-20 $\mu \mathrm{m}$ ) and final (filament length $>20 \mu \mathrm{m}$ ) extension rates were determined from fits to $6 \times 5$ min GFP sum projections. (colors represent individual cells). Bars indicate SD and ${ }^{* * *} p<0.0001$. Figure S5. Distribution of active Cdc42 is not altered during invasive growth. A) Schematic indicating fluorescence signal over the filament long axis. Quantitation of slope of Gaussian farthest from tip in red (Max Slope, in relative units), distance maximum signal to tip $\left(x_{\max }\right.$ in $\left.\mu \mathrm{m}\right)$, and half width half max of the Gaussian farthest from tip in red $\left(\mathrm{x}_{\text {spread }} \mathrm{X}_{\max }\right)$, i.e. the signal spread (Spread in $\mu \mathrm{m}$ ). Signal is denoted by I and distance from tip by $x$. B) Distribution of active $\mathrm{Cdc} 42$ during surface and invasive filamentous growth. Experiment described in Figure 11a and 11b with the mean signal for each cell (colors represents individual cells), normalized to the mean signal for tip Cdc42.GTP in surface growing cells. Bars indicate SD. C) Distribution of active $\mathrm{Cdc} 42$ is not altered upon invasive growth. Relative maximum slope (left), distance from maximum signal to the tip (middle) and spread of signal (right) determined from 6-8 cells, using tailor-made Matlab program. Bars indicate SD; surface and invasive cells were not significantly different. D) Apical and subapical active Cdc42 signals are stable over time. Relative signals from apical and subapical region of sum projections, normalized to maximum invasive subapical signal.

Additional file 2: Movie S1. Invasive growth and penetration into adjacent chamber. Cells grown with indicated stiffness PDMS and followed over time either by DIC optics or fluorescence of labeled with plasma membrane GFP.

Additional file 3: Movie S2. Invasively growing filaments have increased levels of active $\mathrm{Cdc} 42$ at the tip. False colored sum projections of cells expressing CRIB-GFP reporter for active Cdc42.

Additional file 4: Table S1. Strains used in the study [61, 62]. Table S2. Oligonucleotides used in the study. Table S3. Synthesized DNA used in the study.

\section{Acknowledgements}

We thank J. Konopka for reagents, Y. Izmaylov, S. Bogliolo, O. Domenge, and $\mathrm{S}$. Lachambre for the assistance and N. Minc for stimulating discussion.

\section{Authors' contributions}

Conceptualization: X.N., A.S., M.B., and R.A.A. Methodology: C.P., N.K., D.T., S.S., X.N., and R.A.A. Software: S.S. Validation: X.N., A.S., and R.A.A. Formal analysis: C.P., N.K., A.S., and R.A.A. Investigation: C.P., N.K., D.T., and X.N. Data curation: S.S., A.S., and R.A.A. Writing—original draft: M.B. and R.A.A. Writing-review and editing: C.P., N.K., D.T., X.N., A.S., M.B., and R.A.A. Visualization: R.A.A. Supervision: X.N., M.B., and R.A.A. Project administration: X.N., M.B., and R.A.A. Funding acquisition: X.N., A.S., M.B., and R.A.A. All authors read and approved the final manuscript.

\section{Funding}

This work was supported by the CNRS, INSERM, Université Nice-Sophia Antipolis, Université Côte d'Azur and ANR (ANR-15-IDEX-01, ANR-11-LABX-002801, and ANR-16-CE13-0010-01), and EU H2020 (MSCA-ITN- 2015-675407) grants and the Platforms Resources in Imaging and Scientific Microscopy facility (PRISM) and Microscopy Imaging Côte d'Azur (MICA).

\section{Availability of data and materials}

All the data on which the conclusions of the paper are based are presented in the paper and its additional files.
Ethics approval and consent to participate

Not applicable.

\section{Consent for publication}

Not applicable.

\section{Competing interests}

The authors declare that they have no competing interests.

\section{Author details}

${ }^{1}$ Université Côte d'Azur, CNRS, INSERM, Institute of Biology Valrose (iBV), Parc Valrose, Nice, France. ${ }^{2}$ Université Côte d'Azur, CNRS, Institute Physics of Nice (INPHYNI), Ave. J. Vallot, Nice, France. ${ }^{3}$ Present Address: Manchester Fungal Infection Group, School of Biological Sciences, University of Manchester, Manchester, UK. ${ }^{4}$ Present Address: Sorbonne University, CNRS,

Developmental Biology Laboratory (LBDV), Villefranche-sur-mer, France.

Received: 8 April 2020 Accepted: 22 July 2020

Published online: 11 September 2020

\section{References}

1. Campas O, Rojas E, Dumais J, Mahadevan L. Strategies for cell shape control in tip-growing cells. Am J Bot. 2012;99(9):1577-82.

2. Campas O, Mahadevan L. Shape and dynamics of tip-growing cells. Curr Biol. 2009;19(24):2102-7.

3. Lew RR. How does a hypha grow? The biophysics of pressurized growth in fungi. Nat Rev Microbiol. 2011;9(7):509-18.

4. Mendgen $\mathrm{K}$, Hahn $\mathrm{M}$, Deising $\mathrm{H}$. Morphogenesis and mechanisms of penetration by plant pathogenic fungi. Annu Rev Phytopathol. 1996;34: 367-86.

5. Akhtar R, Sherratt MJ, Cruickshank JK, Derby B. Characterizing the elastic properties of tissues. Mater Today (Kidlington). 2011;14(3):96-105.

6. Alonso $\mathrm{J}$, Goldmann $\mathrm{WH}$. Feeling the forces: atomic force microscopy in cell biology. Life Sci. 2003;72(23):2553-60.

7. Mathur AB, Collinsworth AM, Reichert WM, Kraus WE, Truskey GA Endothelial, cardiac muscle and skeletal muscle exhibit different viscous and elastic properties as determined by atomic force microscopy. J Biomech. 2001;34(12):1545-53

8. Dalle F, Wachtler B, L'Ollivier C, Holland G, Bannert N, Wilson D, Labruere C, Bonnin A, Hube B. Cellular interactions of Candida albicans with human oral epithelial cells and enterocytes. Cell Microbiol. 2010;12(2):248-71.

9. Bertuzzi M, Schrettl M, Alcazar-Fuoli L, Cairns TC, Munoz A, Walker LA, Herbst S, Safari M, Cheverton AM, Chen D, et al. The pH-responsive PacC transcription factor of Aspergillus fumigatus governs epithelial entry and tissue invasion during pulmonary aspergillosis. PLoS Pathog. 2014;10(10): e1004413.

10. Basmaciyan L, Bon F, Paradis T, Lapaquette P, Dalle F. Candida Albicans interactions with the host: crossing the intestinal epithelial barrier. Tissue Barriers. 2019;7(2):1612661.

11. Richardson JP, Ho J, Naglik JR. Candida-epithelial interactions. J Fungi (Basel). 2018;4(1).

12. Swidergall M, Filler SG. Oropharyngeal candidiasis: fungal invasion and epithelial cell responses. PLoS Pathog. 2017;13(1):e1006056.

13. Westman J, Hube B, Fairn GD. Integrity under stress: host membrane remodelling and damage by fungal pathogens. Cell Microbiol. 2019;21(4): e13016

14. Wilson D, Naglik JR, Hube B. The missing link between Candida albicans hyphal morphogenesis and host cell damage. PLoS Pathog. 2016;12(10): e1005867.

15. Goyer M, Loiselet A, Bon F, L'Ollivier C, Laue M, Holland G, Bonnin A, Dalle F. Intestinal cell tight junctions limit invasion of Candida albicans through active penetration and endocytosis in the early stages of the interaction of the fungus with the intestinal barrier. PLoS One. 2016;11(3):e0149159.

16. Allert S, Forster TM, Svensson CM, Richardson JP, Pawlik T, Hebecker B, Rudolphi S, Juraschitz M, Schaller M, Blagojevic M, et al. Candida albicansinduced epithelial damage mediates translocation through intestinal barriers. mBio. 2018;9(3):e00915-18.

17. Wachtler B, Citiulo F, Jablonowski N, Forster S, Dalle F, Schaller M, Wilson D, Hube B. Candida albicans-epithelial interactions: dissecting the roles of active penetration, induced endocytosis and host factors on the infection process. PLoS One. 2012;7(5):e36952. 
18. Moyes DL, Wilson D, Richardson JP, Mogavero S, Tang SX, Wernecke J, Hofs S, Gratacap RL, Robbins J, Runglall M, et al. Candidalysin is a fungal peptide toxin critical for mucosal infection. Nature. 2016;532(7597):64-8.

19. Thomson DD, Wehmeier S, Byfield FJ, Janmey PA, Caballero-Lima D, Crossley A, Brand AC. Contact-induced apical asymmetry drives the thigmotropic responses of Candida albicans hyphae. Cell Microbiol. 2015; 17(3):342-54.

20. Martin K, Reimann A, Fritz RD, Ryu H, Jeon NL, Pertz O. Spatio-temporal coordination of RhoA, Rac1 and Cdc42 activation during prototypical edge protrusion and retraction dynamics. Sci Rep. 2016;6:21901.

21. Minc N, Boudaoud A, Chang F. Mechanical forces of fission yeast growth. Curr Biol. 2009;19(13):1096-101.

22. Sevilla MJ, Odds FC. Development of Candida albicans hyphae in different growth media-variations in growth rates, cell dimensions and timing of morphogenetic events. J Gen Microbiol. 1986;132(11):3083-8.

23. Brown XQ, Ookawa K, Wong JY. Evaluation of polydimethylsiloxane scaffolds with physiologically-relevant elastic moduli: interplay of substrate mechanics and surface chemistry effects on vascular smooth muscle cell response. Biomaterials. 2005;26(16):3123-9.

24. Demichelis A, Pavarelli S, Mortati L, Sassi G, Sassi M. Study on the AFM force spectroscopy method for elastic modulus measurement of living cells. J Phys Conf Ser. 2013;459:012050.

25. Xie J, Zhang Q, Zhu T, Zhang Y, Liu B, Xu J, Zhao H. Substrate stiffness-regulated matrix metalloproteinase output in myocardial cells and cardiac fibroblasts: implications for myocardial fibrosis. Acta Biomater. 2014;10(6):2463-72.

26. Wang Z, Volinsky A, Gallant ND. Crosslinking effect on polydimethylsiloxane elastic modulus measured by custom-built compression instrument. J Appl Polym Sci. 2014;131(22):41050.

27. Feng L, Li S, Feng S. Preparation and characterization of silicone rubber with high modulus via tension spring-type crosslinking. RSC Adv. 2017; 7:13130-7.

28. Lorenz MC, Bender JA, Fink GR. Transcriptional response of Candida albicans upon internalization by macrophages. Eukaryot Cell. 2004;3(5):1076-87.

29. McKenzie CG, Koser U, Lewis LE, Bain JM, Mora-Montes HM, Barker RN, Gow NA, Erwig LP. Contribution of Candida albicans cell wall components to recognition by and escape from murine macrophages. Infect Immun. 2010; 78(4):1650-8.

30. Rudkin FM, Bain JM, Walls C, Lewis LE, Gow NA, Erwig LP. Altered dynamics of Candida albicans phagocytosis by macrophages and PMNs when both phagocyte subsets are present. mBio. 2013;4(6):e00810-3.

31. Westman J, Moran G, Mogavero S, Hube B, Grinstein S. Candida albicans hyphal expansion causes phagosomal membrane damage and luminal alkalinization. mBio. 2018;9(5):e01226-18.

32. Vauchelles R, Stalder D, Botton T, Arkowitz RA, Bassilana M. Rac1 dynamics in the human opportunistic fungal pathogen Candida albicans. PLoS One. 2010;5(10):e15400.

33. Silva PM, Puerner C, Seminara A, Bassilana M, Arkowitz RA. Secretory vesicle clustering in fungal filamentous cells does not require directional growth. Cell Rep. 2019;28(8):2231-45 e2235.

34. Desai JV, Cheng S, Ying T, Nguyen MH, Clancy CJ, Lanni F, Mitchell AP. Coordination of Candida albicans invasion and infection functions by phosphoglycerol phosphatase Rhr2. Pathogens. 2015;4(3):573-89.

35. Atilgan E, Magidson V, Khodjakov A, Chang F. Morphogenesis of the fission yeast cell through cell wall expansion. Curr Biol. 2015;25(16):2150-7.

36. Haupt A, Ershov D, Minc N. A positive feedback between growth and polarity provides directional persistency and flexibility to the process of tip growth. Curr Biol. 2018;28(20):3342-51 e3343.

37. Corvest V, Bogliolo S, Follette P, Arkowitz RA, Bassilana M. Spatiotemporal regulation of Rho1 and Cdc42 activity during Candida albicans filamentous growth. Mol Microbiol. 2013;89(4):626-48.

38. Bassilana M, Hopkins J, Arkowitz RA. Regulation of the Cdc42/Cdc24 GTPase module during Candida albicans hyphal growth. Eukaryot Cell. 2005;4(3): 588-603.

39. Delley PA, Hall MN. Cell wall stress depolarizes cell growth via hyperactivation of RHO1. J Cell Biol. 1999;147(1):163-74.

40. Philip B, Levin DE. Wsc1 and Mid2 are cell surface sensors for cell wall integrity signaling that act through Rom2, a guanine nucleotide exchange factor for Rho1. Mol Cell Biol. 2001;21(1):271-80.

41. Bridges AA, Jentzsch MS, Oakes PW, Occhipinti P, Gladfelter AS. Micron-scale plasma membrane curvature is recognized by the septin cytoskeleton. J Cell Biol. 2016;213(1):23-32
42. Delarue M, Poterewicz G, Hoxha O, Choi J, Yoo W, Kayser J, Holt L, Hallatschek O. SCWISh network is essential for survival under mechanical pressure. Proc Natl Acad Sci U S A. 2017;114(51):13465-70.

43. Cullen PJ, Sabbagh W Jr, Graham E, Irick MM, van Olden EK, Neal C, Delrow J, Bardwell L, Sprague GF Jr. A signaling mucin at the head of the Cdc42and MAPK-dependent filamentous growth pathway in yeast. Genes Dev. 2004;18(14):1695-708.

44. Mishra R, van Drogen F, Dechant R, Oh S, Jeon NL, Lee SS, Peter M. Protein kinase $C$ and calcineurin cooperatively mediate cell survival under compressive mechanical stress. Proc Natl Acad Sci U S A. 2017;114(51): 13471-6.

45. Boudaoud A. Growth of walled cells: from shells to vesicles. Phys Rev Lett. 2003;91(1):018104

46. Lockhart JA. An analysis of irreversible plant cell elongation. J Theor Biol. 1965;8(2):264-75.

47. Demoor A, Silar P, Brun S. Appressorium: the breakthrough in Dikarya. J Fungi (Basel). 2019;5(3).

48. Ryder LS, Talbot NJ. Regulation of appressorium development in pathogenic fungi. Curr Opin Plant Biol. 2015;26:8-13.

49. De Silva DD, Crous PW, Ades PK, Hyde KD, Taylor PWJ. Life styles of Colletotrichum species and implications for plant biosecurity. Fungal Biol Rev. 2017;31(3):155-68.

50. Matei A, Doehlemann G. Cell biology of corn smut disease-Ustilago maydis as a model for biotrophic interactions. Curr Opin Microbiol. 2016;34:60-6.

51. Howard RJ, Ferrari MA, Roach DH, Money NP. Penetration of hard substrates by a fungus employing enormous turgor pressures. Proc Natl Acad Sci U S A. 1991;88(24):11281-4.

52. Tanaka E. Appressorium-mediated penetration of Magnaporthe oryzae and Colletotrichum orbiculare into surface-cross-linked agar media. FEMS Microbiol Lett. 2015;362(10):fnv066.

53. Parry DW, Pegg GF. Surface colonization, penetration and growth of three Fusarium species in lucerne. Trans Br Mycol Soc. 1985;85(3):495-500.

54. Bindels DS, Haarbosch L, van Weeren L, Postma M, Wiese KE, Mastop M, Aumonier S, Gotthard G, Royant A, Hink MA, et al. mScarlet: a bright monomeric red fluorescent protein for cellular imaging. Nat Methods. 2017; 14(1):53-6.

55. Zhang C, Konopka JB. A photostable green fluorescent protein variant for analysis of protein localization in Candida albicans. Eukaryot Cell. 2010;9(1): 224-6.

56. Minc N. Microfabricated chambers as force sensors for probing forces of fungal growth. Methods Cell Biol. 2014;120:215-26.

57. Whitesides GM, Ostuni E, Takayama S, Jiang X, Ingber DE. Soft lithography in biology and biochemistry. Annu Rev Biomed Eng. 2001;3:335-73.

58. Schindelin J, Arganda-Carreras I, Frise E, Kaynig V, Longair M, Pietzsch T, Preibisch S, Rueden C, Saalfeld S, Schmid B, et al. Fiji: an open-source platform for biological-image analysis. Nat Methods. 2012;9(7):676-82.

59. Bevington P, Robinson DK. Data reduction and error analysis for the physical sciences. New York: University of California: McGraw-Hill Education; 2003

60. Forster B, Van De Ville D, Berent J, Sage D, Unser M. Complex wavelets for extended depth-of-field: a new method for the fusion of multichannel microscopy images. Microsc Res Tech. 2004;65(1-2):33-42.

61. Wilson RB, Davis D, Mitchell AP. Rapid hypothesis testing with Candida albicans through gene disruption with short homology regions. J Bacteriol. 1999;181(6):1868-74.

62. Bassilana M, Blyth J, Arkowitz RA. Cdc24, the GDP-GTP exchange factor for Cdc42, is required for invasive hyphal growth of Candida albicans. Eukaryot Cell. 2003;2(1):9-18.

\section{Publisher's Note}

Springer Nature remains neutral with regard to jurisdictional claims in published maps and institutional affiliations. 